msh-mss Mathématiques et sciences humaines

167| Automne 2004

Varia

\title{
Pierre-François Verhulst et la loi logistique de la population
}

Pierre-François Verhulst and the logistic law of population

\section{Bernard Delmas}

\section{(2) OpenEdition \\ Journals}

Édition électronique

URL : http://journals.openedition.org/msh/2893

DOI : $10.4000 / \mathrm{msh} .2893$

ISSN : 1950-6821

Éditeur

Centre d'analyse et de mathématique sociales de l'EHESS

Édition imprimée

Date de publication : 1 septembre 2004

ISSN : 0987-6936

Référence électronique

Bernard Delmas, « Pierre-François Verhulst et la loi logistique de la population », Mathématiques et sciences humaines [En ligne], 167 | Automne 2004, mis en ligne le 10 mars 2006, consulté le 23 juillet 2020. URL : http://journals.openedition.org/msh/2893 ; DOI : https://doi.org/10.4000/msh.2893

〔c École des hautes études en sciences sociales 


\title{
DOSSIER «! DÉMOGRAPHIE MATHÉMATIQUE!»
}

\author{
$-5-$
PIERRE-FRANÇOIS VERHULST ET LA LOI LOGISTIQUE DE LA POPULATION ${ }^{1}$

\author{
Bernard DELMAS ${ }^{2}$
}

\begin{abstract}
RÉSUMÉ - Avec les progrès réalisés par l'analyse démographique, les premières tentatives de formalisation de la dynamique des populations semblent avoir perdu presque tout intérêt théorique et pratique. Mais, si les recherches privilégiant l'approche globale - où s'étaient illustrés Quetelet et son élève Verhulst dans les années trente du XIXe siècle, puis Pearl et Reed dans les années vingt du siècle suivant - sont tombées en déshérence en ce qui concerne la prévision de croissance des populations humaines, elles représentent cependant un épisode très significatif de l'histoire de la "mathématisation » des sciences sociales.

MOTS CLÉS - Verhulst, Quetelet, Malthus, Raymond Pearl, Loi logistique, Dynamique des populations, Modèles démographiques, Prévisions démographiques.

SUMMARY!- Pierre-François Verhulst and the logistic law of population With the progress made by demographic analysis, the first attempts to formalize the dynamics of populations seem to have lost much of their theoretical and practical interests. But, if researches favouring the global approach - through which Quetelet and his disciple Verhulst became famous in the 1830s, then Pearl and Reed in the 1920s - have become obsolete, as far as prediction of human populations growth is concerned, they nevertheless represent a very significant landmark in the history of the "mathematisation" of the social sciences.
\end{abstract}

KEY-WORDS!- Verhulst, Quetelet, Malthus, Raymond Pearl, Logistic law, Population dynamics, Demographical models, Demographical previsions.

\footnotetext{
${ }^{1}$ Article reçu le!10 juin 2003, révisé le!26 avril 2004, accepté le 14 mai 2004.

Une première version de ce texte avait été présentée au Colloque de l'Association Charles Gide!: Modèles formels et théorie économique organisé à la Sorbonne par le Professeur Annie L. Cot en septembre 1999, une seconde au Séminaire d'histoire du calcul des probabilités et de la statistique de Marc Barbut et Éric Brian (EHESS, Centre Alexandre Koyré et Centre d'analyse et de mathématique sociales) publiée dans Les Cahiers du CAMS n 186 en mars 2000. On trouvera sur le site http://www.ifresi.univlille1.fr/economistes.html, les textes de Verhulst étudiés ici.

${ }^{2}$ CLERSÉ, Université de Lille I, Faculté des sciences économiques et sociales, bât. S.H. 2, 59655 Villeneuve-d'Ascq cedex, bernard.delmas@univ-lille1.fr
} 


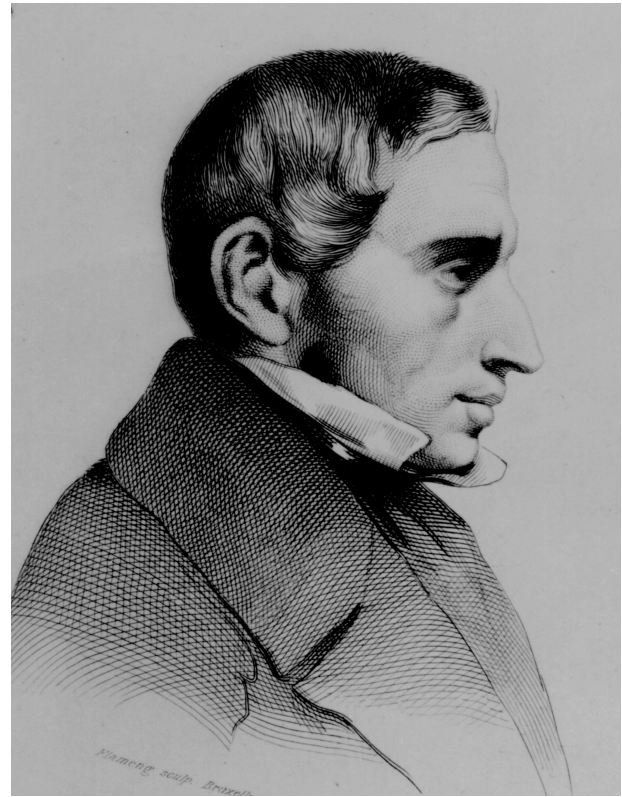

Figure 1. Pierre-François Verhulst ${ }^{3}$ (1804-1849)
La population forme, je dirai presque la substance autour de laquelle se réalisent et se développent tous les phénomènes de l'économie sociale. C'est par elle que tout s'agite et s'accomplit dans le monde économique. Instrument principal de la production, c'est à son bénéfice que s'opère la distribution de la richesse nationale : elle est à la fois le but et le moyen. Aussi la science économique pourrait-elle se résumer tout entière dans la science de la population; elle en est, du moins, le commencement et la fin [Rossi, 1840] $]^{4}$.

Le bicentenaire de Pierre-François Verhulst (né à Bruxelles le 28 octobre 1804) semble avoir suscité à ce jour peu de manifestations d'intérêt de la part de la communauté scientifique, si l'on excepte l'intéressant colloque réuni en septembre dernier à l'École royale militaire de Belgique, où Verhulst avait occupé la première chaire d'analyse mathématique. Encore ce colloque était-il consacré à un héritage assez lointain de la fameuse loi de Verhulst, puisqu'il portait essentiellement sur l'actualité de la théorie du chaos.

Les premières tentatives de formalisation de la dynamique des populations ont manifestement perdu beaucoup de leur intérêt pour les démographes contemporains!: les recherches privilégiant l'approche globale, où s'étaient illustrées Quetelet et Verhulst dans les années 30 du XIX ${ }^{\mathrm{e}}$ siècle, puis Pearl et Reed dans les années 20 du siècle suivant, sont tombées en déshérence, au moins en matière de prévision de croissance des populations humaines ${ }^{5}$. Peu connues et peu étudiées ${ }^{6}$, elles représentent cependant

\footnotetext{
${ }^{3}$ Portrait gravé par Flameng, figurant dans la «!Notice sur Pierre-François Verhulst![...]!» de Quetelet dans l'Annuaire de l'Académie Royale pour 1850.

${ }^{4}$ Pellegrino Rossi, Cours d'économie politique, p. 394-395. Citation placée par Verhulst en exergue de son «!Deuxième mémoire...», p. 1. Voir la bibliographie in fine.

${ }^{5}$ Par contre, la recherche de modèles mathématiques de dynamique des populations animales plus réalistes continue de mobiliser les spécialistes de bio-mathématique et d'écologie théorique, dans la lignée des travaux fondateurs publiés dans l'entre-deux guerres par Lotka, Volterra et d'Ancona. On en trouvera un aperçu très suggestif et les principales références dans l'article de Jean Mawhin!: «!les héritiers de Pierre-François Verhulst!: une population dynamique!» (à paraître dans le Bulletin de la Classe des Sciences de l'Académie Royale de Belgique).

${ }^{6}$ Voir cependant l'Histoire de la démographie de J. et M. Dupâquier!; dans la revue Population, l'article!: «!La première découverte de la fonction logistique!» de M. Schtickzelle [1981], ainsi que pour des développements plus récents!: Population forecasting 1895-1945, de Henk de Gans!; «!Les mathématiques de la population de Lambert à Lotka!» de Jacques Véron, Mathématiques et sciences humaines, $\mathrm{n}^{\circ}$ 159, vol. 40, 2002, et l'article de J. Mawhin déjà cité.
} 
un épisode très significatif de l'histoire de la «!mathématisation du réel!» ${ }^{7}$ dans les sciences sociales.

Quand il n'est pas complètement négligé dans les ouvrages courants d'histoire des idées démographiques, on ne retient généralement de Verhulst que l'invention, en 1844, de l'équation de la courbe logistique, cette fameuse courbe «!en $S$ » aplati -!ou «!sigmoïde!»! - que 1'on oppose souvent à l'exponentielle «!de Malthus!», caractéristique de la croissance géométrique de la population?.

En fait, comme nous le verrons plus loin, la loi de Verhulst n'a en aucun cas été conçue comme concurrente à la loi de Malthus. Tout au contraire, il s'agit pour Verhulst de dépasser l'hétérogénéité de l'exposé de l'auteur de l'Essai sur le principe de population qui combine!:

- un exposé, illustré par un exemple mathématique, des tendances à la croissance en série géométrique de la population et en série arithmétique des subsistances!;

- et une description purement littéraire des obstacles -!préventifs et destructifs!- qui s'opposent à la croissance indéfinie de la population.

Son objectif est en effet de formuler en termes analytiques la «!loi de la nature!» ${ }^{10}$ gouvernant la croissance démographique ou, au moins, de bâtir, sur la base des intuitions de Malthus, un modèle homogène en cherchant à «!appliquer le calcul au principe de population!» ${ }^{11}$.

En tentant de définir les notions de «!population normale!» et de «!population maximale!», tout en reliant par une fonction le rythme de croissance au niveau de la «!population surabondante!», Pierre-François Verhulst, brûlant de vitesse son maître Adolphe Quetelet, est parvenu à bâtir le premier véritable modèle démo-économique. Après une éclipse ${ }^{12}$ de près de 80 ans, ce modèle, allait être «!redécouvert!» en 1920 par Raymond Pearl et Lowell Reed ${ }^{13}$, puis servir longtemps d'outil essentiel pour la

\footnotetext{
${ }^{7}$ Pour reprendre le titre de l'œuvre de Giorgio Israel dont la lecture nous a beaucoup inspiré lors de la rédaction de cet article.

${ }^{8}$ On pourra trouver d'autres modèles de type sigmoïde par exemple dans La régression, nouveaux regards sur une ancienne méthode statistique, de R. Tomassone et al. [1983] et «!Modélisation de croissances décrites par une courbe sigmoïde!» J. Bair et al. [1998].

${ }^{9}$ Voir par exemple Delevsky!: «!Verhulst a prévu, le premier, que, contrairement à la doctrine de Malthus, l'accroissement de la population diminuerait graduellement, comme effet de la diminution de la natalité, et que le mouvement de la population tendrait vers un état stationnaire, une certaine limite numérique ne pouvant être dépassée », «Une formulation mathématique![...] », Metron, n 4, 1928, p. 88.

${ }^{10}$ Ibidem, p. 17.

11 Verhulst!: «!Recherches mathématiques sur la loi d'accroissement de la population!» [Premier Mémoire], 1845, p. 4.

${ }^{12}$ Verhulst reste cependant cité de manière épisodique, par exemple par Liagre dans son Calcul des probabilités de 1879 ou dans la bibliographie de von Fircks (1898).

${ }^{13}$ Voir infra, Bibliographie, section C. Dans son ouvrage de référence!: Modeling Nature (1985) p.!66, Sharon E. Kingsland signale que la première mention de Verhulst (sans références) par Pearl se trouve dans un article de The Scientific Monthly de 1921!: «!The Biology of Death -!Natural Death, Public Health and the Population Problem!». On y lit, dans une note p.!206!: «!The mathematical hypothesis here dealt with is essentially the same as that of Verhulst put forth in 1844 ![...]!». Selon S. Kingsland, il est sans doute exact que Pearl n'a pas eu connaissance auparavant des travaux de Verhulst, mais il est certain qu'il a été influencé par ceux du biochimiste Thorburn Brailsford Robertson -!publiés en 1908!- où celuici «!invente!» une courbe dite «!autocatalytique!» qui ressemble en tout point à la «!logistique!». Les recherches de Verhulst et de Quetelet sur la croissance des populations sont-elles restées inconnues de Robertson lui-même!? Celui-ci n'en dit rien dans ses articles mais, curieusement, il y utilise certaines des
} 
construction des perspectives démographiques, et alimenter pendant des décennies le débat sur l'équilibre population-ressources ${ }^{14}$.

\title{
1. L'ANALOGIE MÉCANIQUE DE QUETELET ${ }^{15}$
}

Les travaux de Verhulst ne peuvent prendre toute leur signification que si on les compare à ceux de Quetelet, ou tout au moins, dans la masse énorme des publications de ce dernier, à ses thèses essentielles sur la population. Ceci se justifie non seulement parce que Verhulst était le principal disciple de l'académicien, et parce que ses recherches sur la population résultaient directement d'une «!commande!» de Quetelet, mais surtout, nous le verrons, parce que leurs approches épistémologiques sont complètement différentes, voire même antagonistes. Une évocation rapide des positions de Quetelet permettra de mieux comprendre les enjeux de ce débat.

Dès 1835, dans la première édition de sa «!Physique sociale!», Adolphe Quetelet avait fixé les grandes lignes de sa conception du fonctionnement du corps social et des conséquences qu'il en tirait pour son programme de recherche!:

- Le bien-être ou la pauvreté de la population ne résulte pas de l'effet erratique d'une fatalité imprévisible mais de l'action de lois qui peuvent être décrites par la science sociale et servir de base aux prévisions.

- Ces lois s'imposent certes au corps social, mais les hommes les connaissant peuvent les infléchir par leur action et faire, du bien-être social, un objectif. «!Comme membre du corps social, [l'homme] subit à chaque instant la nécessité des causes et leur paie un tribut régulier!; mais comme homme, usant de toute l'énergie de ses facultés intellectuelles, il maîtrise en quelque sorte ces causes, modifie leurs effets, et peut chercher à se rapprocher d'un état meilleur» ${ }^{16}$.

- Cet objectif est compromis en cas de déséquilibre entre la croissance de l'effectif global de la population et celle des moyens de subsistance, tel est donc le problème crucial qu'il faut éclairer en construisant une science de la population.

- La question de la structure de la population est un problème second mais important car cette structure influe, à population égale, sur le niveau du bien-être.

\begin{abstract}
Le programme est clairement construit : [L'accroissement de la population] est plus ou moins rapide, et provient, soit d'un excès des naissances sur les décès, soit des immigrations. Il annonce en général un état de bien-être et des moyens d'existence supérieurs aux besoins de la population actuelle. Si l'on approche de cette limite ou si on la dépasse, bientôt l'état de croissance s'arrête et fait place à un état contraire.
\end{abstract}

données anthropométriques publiées par Quetelet en précisant à plusieurs reprises qu'il les cite d'après les «!British Association Anthropometric Commitees Reports!». Le mystère de la filiation est donc reporté en amont, s'il n'est pas éclairci. Relevons pour terminer qu'à partir de ces premiers travaux, Robertson développa toute une théorie de la croissance comme phénomène d'autocatalyse et qu'il crut même avoir découvert l' «!autocatalyseur!» en cause et le fit breveter. Bon prince, il fit don des droits à l'Université de Californie, sans doute pour l'aider à croître..

${ }^{14}$ Voir sur ce point de Gans!: Population forecasting, op. cit. et L'intelligence démographique de PaulAndré Rosental, p. 178 sq.

${ }^{15}$ Je tiens à remercier ici chaleureusement M. De Paepe, attaché scientifique à l'Académie Royale de Belgique (et auteur de l'excellent Inventaire des Archives de l'Académie), ainsi que son adjoint M. Maldague, pour la très grande gentillesse et disponibilité dont ils ont fait preuve lors de mes recherches dans le fonds Quetelet de l'Académie.

${ }^{16}$ A. Quetelet, Sur l'homme et le développement de ses facultés ou essai de physique sociale, 1835 [cité ici d'après la réédition de 1991]. «!Introduction!», p. 37-38. 
Il est donc intéressant de rechercher combien les différents pays ont de population, quels sont les moyens de subsistance et les degrés d'accroissement de ces populations, et d'assigner la limite qu'ils peuvent atteindre sans danger.

Il se présente ensuite la question de savoir comment les populations se composent, si leurs éléments constitutifs sont avantageusement distribués, et contribuent d'une manière plus ou moins efficace au bien-être de l'ensemble ${ }^{17}$.

Quant au schéma de base de l'analyse des interactions entre population et subsistances, il est directement repris de Malthus, que Quetelet a bien connu et avec qui il a contribué à une certaine institutionnalisation de la statistique en Grande-Bretagne, avant d'étendre le projet au niveau international. Le point fort de cette collaboration avait été la troisième réunion de l'Association Britannique pour l'Avancement des Sciences en 1833 à Cambridge ${ }^{18}$ et, comme il ne manquait pas de le signaler à ce propos!:

Malthus professait une grande estime pour la Belgique ; il regardait ce pays comme méritant au plus haut degré l'étude du savant, à cause de sa position circonscrite, de sa diversité comme terrain agricole et manufacturier etc... Il m'avait confié à cet égard une note qui fut remise au gouvernement et que j'ai regretté de voir négligée $[\ldots]^{19}$.

Quetelet, avec Malthus, Babbage et quelques autres, avaient été à l'initiative de l'ouverture, au sein de la conférence d'une section spéciale consacrée à la statistique ${ }^{20}$. La présidence fut proposée à Malthus qui suggéra à sa place Babbage, et Quetelet joua un rôle très actif lors des réunions du comité. Il put y mettre en avant la qualité des travaux statistiques entrepris en Belgique et proposer pour son petit pays -!fort du soutien de Malthus!- une sorte de statut de «!laboratoire démographique!» de l'Europe!:

Peu de pays, par leur position, par la nature de leurs limites et la tenue des registres de l'état-civil, méritent autant que le nôtre d'être étudiés sous le rapport de la population [...]. Les renseignements que je fus à même de présenter [...] et l'assurance que je crus pouvoir donner que notre

\footnotetext{
${ }^{17}$ Ibid., Livre $1^{\text {er }}$, Section VII-1 «!De la population et de ses accroissements!», p.!247-248.

${ }^{18}$ Voir la présentation de la $2^{\mathrm{e}}$ éd. de!:! «!Physique sociale, sur 1'homme et le développement de ses facultés!», par Quetelet, Bulletins de l'Académie [...], 1868, p.!496-502 et ses «!Notes extraites d'un voyage en Angleterre [...]!», Correspondance mathématique et physique, t.!8, 1835, p.!1-16.

${ }^{19}$ Quetelet, présentation de la $2^{\mathrm{e}}$ éd. de!:! «!Physique sociale », Bulletins de l'Académie Royale, 1868, op. cit., p.!499. Cette note manuscrite de Malthus est conservée dans les Archives Quetelet de l'Académie royale de Bruxelles [liasse $n^{\circ}$ 1697], elle a été publiée dans l'ouvrage de Patricia James, Population Malthus, p.!446. La note fixe le programme de collecte statistique de base, souhaitable pour l'analyse démographique et l'étude du rapport entre population et subsistances, elle est titrée «Desiderata »!:

«!The number of births to each marriage in the course of its duration.!/ The proportion of the born wich lives to marry.!/ The number of living children to each marriage.!/ The money wages of labour manufacturing and agricultural in the different provinces, particularly the wages of common day labour in agricultural employment.!/ The quantity of wheat which the wages of such day labour will purchase in ordinary times.!/ The ordinary prices of different kinds of grain.!/ The ordinary food of the common daylabourer!» [la rature est de la main de Malthus].

${ }^{20}$ La création de cette section ne s'était d'ailleurs pas faite sans problèmes, le président de l'Association, le géologue Adam Sedgwick, craignant que, pour certains des membres, la statistique soit considérée comme «!ill-fitted to a Society formed only for the promotion of natural science!». Il avait consenti finalement en se justifiant par une définition élargie de la science conçue comme «!the consideration of all subjects, whether of pure or mixed nature, capable of being reduce to measurement and calculation. [...] All phenomena capable of being brought under the semblance of a law are legitimate objects of our inquiries!» [cité par P. James, op. cit., p.!446, c'est nous qui soulignons, B.D.].
} 
gouvernement se prêterait volontiers à faire toutes les recherches dont la science aurait besoin, porta à croire qu'on pourrait avec succès prendre notre royaume comme offrant toutes les conditions désirables pour servir de sujet d'étude $e^{21}$.

Mais Quetelet jugeait trop restreint le rôle assigné au comité, il fit donc pression sur son président Babbage pour la création de la Statistical Society of London, dont l'objet, selon la résolution rédigée par Malthus devait être "the collection and classification of all facts illustrative of the present condition and prospects of society"22. La première réunion eu lieu le 15 Mars 1834 et la société survit encore aujourd'hui sous le nom de Royal Statistical Society, qu'elle porte depuis 1877.

Le point de départ de l'analyse de la croissance de la population est donc directement repris de Malthus!:

Il paraît incontestable que la population crôtrait selon une progression géométrique, s'il ne se présentait aucun obstacle à son développement.

Les moyens de subsistance ne se développent point aussi rapidement, et, selon Malthus, dans les circonstances les plus favorables à l'industrie, ils ne peuvent jamais augmenter plus vite que selon une progression arithmétique.

Le grand obstacle à la population est donc le manque de nourriture, provenant de la différence des rapports que suivent ces deux quantités dans leurs accroissements respectifs.

Quand une population, dans son développement, est parvenue au niveau de ses moyens de subsistance, elle doit s'arrêter à cette limite par la prévoyance des hommes ; ou si elle a le malheur de la franchir, elle s'y trouve forcément ramenée par un excès de mortalité.

Les obstacles à la population peuvent donc être rangés sous deux chefs. Les uns agissent en prévenant l'accroissement de la population, et les autres en la détruisant à mesure qu'elle se forme. La somme des premiers compose ce que l'on peut appeler l'obstacle privatif; celle des seconds l'obstacle destructif $^{23}$.

Comme on le voit, Quetelet a parfaitement compris la thèse de Malthus. Il ne commet pas l'erreur, si fréquente dans les gloses de l'Essai sur le principe de population, consistant à réduire cette thèse à la comparaison des deux progressions, arithmétique et géométrique, en ignorant le point essentiel, c'est-à-dire la description des obstacles à la croissance de la population. Sa critique est donc d'autant plus pertinente et constructive.

Elle consiste tout d'abord à nuancer la loi de la croissance arithmétique des subsistances en indiquant que l'échange des produits industriels permet à certains pays de compenser un déficit éventuel de production agricole!:

Si une population consomme tous les produits de la terre qu'elle habite, elle peut, par l'échange de ses autres produits, suppléer à ce qui lui manquerait, dans le cas où elle recevrait de nouveaux accroissements : ainsi, la multiplicité des machines, en secondant les travaux de l'homme, en Angleterre, a permis aux moyens de subsistance de prendre, depuis le

\footnotetext{
${ }^{21}$ Quetelet, Correspondance mathématique et physique, t. 8, 1835, p. 15.

${ }^{22}$ Cité par P. James, Population Malthus, op. cit., p. 449.

${ }^{23}$ A. Quetelet, Sur l'homme [...], op. cit., p. 248-250. Quetelet précise ici, en note!: «!Dans la manière de voir que j'ai adoptée, l'obstacle destructif appartient généralement aux forces naturelles, et l'obstacle privatif aux forces perturbatrices de l'homme!».
} 
commencement de ce siècle, un accroissement qui me semble beaucoup plus rapide que celui de la progression arithmétique. On ne pourrait cesser de regarder les produits industriels à l'égal des produits de l'agriculture que quand les échanges seraient devenus impossibles par un développement trop grand de la population sur les différents points du globe $^{24}$.

La limite des subsistances est donc repoussée très loin, elle n'est pas un obstacle «!tant que les choses n'en seront pas venues au point où toutes les parties du globe seront livrées à la culture!» ${ }^{25}$.

Mais la principale critique est d'un tout autre ordre!: si Malthus a bien analysé les principaux obstacles à l'accroissement de la population et déterminé «!la limite qu'elle ne saurait dépasser sans s'exposer aux plus grands préjudices!», ni lui ni ses successeurs n'ont établi «!le mode d'action des obstacles!» ou plus précisément, «!la loi en vertu de laquelle ils agissent!». Ainsi «!on n'a pas donné les moyens de transporter la théorie de la population dans le domaine des sciences mathématiques, auquel elle semble spécialement devoir appartenir!» et «!on s'est peut-être exagéré les dangers que courait la société, en ne trouvant pas assez de garanties dans l'action des obstacles contre le mal dont la vitesse effrayante suivait la progression géométrique $»^{26}$.

Ce point est tellement essentiel pour Quetelet qu'il avait inscrit en exergue de son traité Sur l'homme une phrase extraite de l'Essai philosophique sur les probabilités de Laplace «!Appliquons aux sciences politiques et morales la méthode fondée sur l'observation et le calcul, méthode qui nous a si bien servi dans les sciences naturelles!». Et il insiste en citant -!dans le même passage du chapitre sur la population et ses accroissements!- un de ses textes de 1827!: «!Il est à remarquer [...] que plus les sciences physiques ont fait de progrès, plus elles ont tendu à rentrer dans le domaine des mathématiques, qui est une espèce de centre vers lequel elles viennent converger. On pourrait même juger du degré de perfection auquel une science est parvenue, par la facilité plus ou moins grande avec laquelle elle se laisse aborder par le calcul! ${ }^{27}$.

Ce thème est repris avec insistance par Quetelet dans une lettre au Ministre de l'Intérieur de Belgique Sylvain Van de Weyer de 1834, présentant son nouvel ouvrage $^{28}$ !:

Je me fais peut-être illusion, mais j'ai lieu de croire que le travail fera plaisir [...] la partie la plus curieuse du travail sera, je crois, la théorie de la population. Je suis parvenu à la transporter entièrement dans le domaine des sciences exactes, au moyen de deux théorèmes dont l'un m'appartient. J'ai soumis ma théorie à l'épreuve de l'expérience et les résultats calculés s'accordent parfaitement avec les résultats observés. Je ne fais au fond que montrer que ce qui est admis par les principaux économistes et ce qui se trouve dans les ouvrages de Malthus peut s'énoncer d'une manière plus précise et se formuler de manière plus élégante.

On pourra résoudre les grands problèmes des mouvements de la population comme ceux des mouvements des corps célestes; et ce qu'il y a de plus remarquable, c'est l'étonnante analogie qui existe entre les formules qui

\footnotetext{
${ }^{24}$ Ibidem, note 1, p. 248.

${ }^{25}$ Ibidem.

${ }^{26}$ Op. cit., p. 249-250.

${ }^{27}$ Voir note, ibidem, p. 249-250. Texte extrait de la séance inaugurale d'un cours public d'histoire des sciences.

${ }^{28}$ Source!: Papiers Van de Weyer, ${ }^{\circ} 247$, Archives générales du royaume, Bruxelles. Voir note suivante.
} 
servent à ces calculs. Je crois avoir réalisé en partie ce que j'ai dit depuis longtemps sur la possibilité de faire une mécanique sociale comme l'on a une mécanique céleste; de formuler les mouvements du corps social comme on a formulé les mouvements des corps célestes et d'en reconnaître toutes les propriétés et les lois conservatrices. Vous allez peut-être me taxer de présomption ou croire que ma raison a subi quelqu'anomalie; je dois cependant vous rassurer à cet égard. Je pense même que quand on verra combien cette théorie est simple et combien il fallait peu d'efforts pour la mettre sur pied, on se demandera s'il y avait quelque mérite à le faire ${ }^{29}$.

Le même thème reviendra encore dans la conclusion de sa présentation de la deuxième édition de sa Physique sociale en $1868^{30}$ !:

Tandis que le savant cherchait avec le plus vif empressement les lois physiques et mécaniques qui régissent les plantes et les animaux, tandis qu'il s'occupait avec les soins les plus assidus de tout ce qui peut contribuer à leur développement, le statisticien devait nécessairement avoir en vue si les divers développements de l'homme n'étaient pas assujettis à des lois dont on pût rendre compte; si l'homme, par exemple, croissait en hauteur selon des principes formulés d'avance; si son poids, sa force, sa vitesse, étaient soumis à des lois appréciables; si ses qualités intellectuelles et morales étaient également réglées avec mesure et prudence comme tout ce qui s'observe dans la nature. [...] On peut dire même, contrairement à ce que l'on avait cru d'abord, que les phénomènes de l'homme s'accomplissent avec infiniment plus d'harmonie que ceux des plantes et des animaux ${ }^{31}$.

Une telle attitude est une illustration presque parfaite de la tendance au «!réductionnisme physique!», ou plus exactement ici à l'!«!analogie mécanique!» dont les raisons ont été si bien décrite par Giorgio Israel!:

L'étude mathématique de la nature a débuté dans le contexte de la science du
mouvement (la mécanique), de l'astronomie et, plus généralement, de l'étude
des phénomènes physiques. [...] Dans la formation de la mécanique et plus
généralement de la physique, les mathématiques ont eu un rôle constitutif.
On comprend ainsi non seulement que la physique est liée d'une façon
indissoluble aux images mathématiques, mais qu'en outre, dans les
descriptions mathématiques des phénomènes, les images physiques sont les
images privilégiées et, pour ainsi dire, "naturelles". D’où le recours
"spontané" à l'analogie mécanique, car la mécanique a été, tant du point de

${ }^{29}$ Lettre de Quetelet au Ministre de l'Intérieur de Belgique, Sylvain Van de Weyer, du 22 août 1834, à propos de son livre Sur l'homme [...], reproduite in Académie Royale, Adolphe Quetelet, 1796 - 1874. Exposition documentaire [...], 1974, p. 91-92.

Quetelet poursuit!: J'ai montré toute cette affaire à MM. Plateau et Verhulst qui m'ont fait l'honneur de regarder cela comme très raisonnable; le dernier même m'a dit qu'il était parvenu à un résultat semblable; et je crois que c'est là ce que me diront la plupart des personnes qui me feront l'honneur de me lire. Cependant je ne dois pas trop me flatter à cet égard; car, comme le dit fort bien Say, on n'a commencé à révoquer en doute l'existence des obstacles à la population que depuis qu'elle a été si bien démontrée par Malthus.

${ }^{30}$ Quetelet, Bulletins de l'Académie [...], 1868, op. cit., p.!500-503.

${ }^{31}$ Ibidem. Quetelet poursuit en évoquant la question très controversée du déterminisme, que nous n'aborderons pas ici!: Ce qui m'a singulièrement étonné, c'est que, au premier abord, des savants de profond mérite ne virent, dans ces recherches, que des essais qui tendaient à mettre l'homme au-dessous de sa valeur, en astreignant ses actions mêmes à devenir en quelque sorte mécaniques. J'ose croire tout le contraire : ici encore, le champ intellectuel de l'homme s'étend pendant que la partie matérielle perd de son empire; son savoir pénètre les plus grandes profondeurs des cieux et s'en explique les mouvements, qui lui étaient inconnus d'abord, tandis que ses facultés physiques, tout en se resserrant, agissent avec plus d'ordre dans ce vaste ensemble. 
vue historique que du point de vue conceptuel, le noyau de formation de la physique ${ }^{32}$.

Pour des disciples de Laplace, tels que Quetelet, «!la seule façon de bâtir une "vraie" science mathématique du monde moral était donc d'imiter strictement la mécanique, tant du point de vue des méthodes que des techniques mathématiques ${ }^{33}$.

Ce recours «!spontané!» à l'analogie physique est clairement invoqué par Quetelet lorsqu'il expose sa théorie mathématique de la croissance de la population et des obstacles qu'elle rencontre!:

La théorie de la population peut se réduire aux deux principes suivants, que je regarde comme devant servir désormais de principes fondamentaux à l'analyse du développement de la population et des causes qui l'influencent.

La population tend à croître selon une progression géométrique.

La résistance, ou la somme des obstacles à son développement, est, toutes choses égales d'ailleurs comme le carré de la vitesse avec laquelle la population tend à croître ${ }^{34}$.

Mais le terme d'analogie est presque trop faible ici, encore que Quetelet l'utilise lui-même. Pour lui en effet, la loi de population ne fonctionne pas comme une loi physique, c'est réellement une loi physique!:

Les obstacles à la vitesse d'accroissement d'une population, agissent donc réellement comme la résistance qu'opposent les milieux au mouvement des corps qui les traversent. Cette extension d'une loi de la physique [...] offre un exemple nouveau des analogies qu'on trouve, dans bien des cas, entre les lois qui règlent les phénomènes matériels et ceux qui sont relatifs à l'homme. De sorte que, des deux principes que je prends pour bases de la théorie mathématique de la population, l'un est généralement admis par tous les économistes, et il ne semble guère susceptible d'être contesté, et l'autre a été vérifié dans toutes les applications où l'on avait à considérer le mouvement et des obstacles agissant d'une manière continue ${ }^{35}$.

La fin de la citation éclaire le processus de raisonnement de Quetelet. C'est manifestement le modèle physique de la résistance aérodynamique ${ }^{36}$ qui a fourni à l'auteur l'idée de sa formule de freinage de la croissance de la population, celle-ci n'étant sans doute considérée que comme un cas particulier d'un processus rencontrant «!des obstacles agissant d'une manière continue!». Consciemment ou non, le mathématicien et astronome Quetelet devait avoir cette formule en tête quand il recherchait l'expression mathématique de la loi de résistance même s'il est vrai, comme

\footnotetext{
${ }^{32}$ G. Israel, op. cit., p. 11.

${ }^{33}$ G. Israel, ibidem, p. 161. Israel signale une attitude similaire de la part de Walras!: «!Façonner la science économique sur le modèle de la science la plus parfaite, n'était-ce pas la meilleure garantie de la validité de l'approche choisie!» (Ibidem, p. 320).

${ }^{34}$ Sur l'homme, op. cit., p. 250.

${ }^{35}$ Ibidem, p.!250-251 (C'est nous qui soulignons). Quetelet reprend plus loin, dans la même veine, une autre métaphore physique, celle du nuage qui, malgré les aléas atmosphériques, «!revient toujours à une certaine hauteur moyenne, dépendante de sa constitution et de l'obstacle qu'il éprouve à sa chute par la résistance de l'air!».

${ }^{36}$ La résistance aérodynamique s'écrit, rappelons-le!: $\mathrm{R}_{\mathrm{a}}=1 / 2 \cdot \mu . \mathrm{k} \cdot \mathrm{v}^{2}$ (où $\mu$ représente la masse volumique de l'air, $\mathrm{k}$ un coefficient dépendant de la surface frontale du véhicule et de sa résistance aérodynamique et $\mathrm{v}$ sa vitesse). Pour une formulation contemporaine de Quetelet de ce principe, v. par exemple Poisson, op. cit., tome!I, p.!242-243.
} 
il le prétend que!: «!pour chercher à remplir une lacune aussi importante [dans la thèse de Malthus], je me suis livré à des recherches nombreuses dont il serait superflu de présenter ici les détails! ${ }^{37}$.

La comparaison avec les données disponibles étant en tout cas probante aux yeux de Quetelet, il s'autorise à conclure!:

Ainsi, quand une population peut se développer librement et sans obstacles,
elle crô̂t selon une progression géométrique ; si le développement a lieu au
milieu d'obstacles de toute espèce qui tendent à l'arrêter, et qui agissent
d'une manière uniforme, c'est-à-dire si l'état social ne change point, la
population [...] tend de plus en plus à devenir stationnaire. Il en résulte donc
que la population trouve, dans sa tendance même à croître, les causes qui
doivent prévenir les funestes catastrophes qu'on pourrait craindre par un
trop-plein, si je puis m'exprimer ainsi, amené d'une manière brusque, et
devant lequel toute la prudence humaine viendrait échouer. L'expérience
même de notre vieille Europe prouve très bien que les populations arrivent à
leur état d'équilibre, ou croissent ou rétrogradent en suivant généralement
une loi de continuité. La limite qu'elles ne peuvent dépasser, est variable de
sa nature, et se trouve réglée par la quantité des subsistances ; jamais les
populations ne peuvent se développer avec une rapidité assez grande pour
venir brusquement se heurter contre cette limite ; les obstacles qui naissent
dans son voisinage sont trop nombreux pour ne pas rendre généralement
impossible un choc violent» ${ }^{38}$.

Quetelet en déduit d'ailleurs une conclusion intéressante pour l'analyse économique comparative!: la croissance de la civilisation, de la force productive d'un pays se traduisant par une augmentation de sa population stationnaire, la densité de population ${ }^{39}$ pourrait être considérée comme «!la meilleure mesure de la production et de l'industrie!» ${ }^{40}$ d'une nation. Mais Quetelet nuance aussitôt cette règle en faisant intervenir deux dimensions à prendre en compte dans les comparaisons entre pays!:

- «!un coefficient constant, dépendant de ce qui est nécessaire à un individu de chaque nation pour subvenir à ses besoins!» ${ }^{41}$, c'est-à-dire ce que nous appellerions un indice différentiel de niveau de vie.

- le degré d'inégalité dans la répartition des richesses, car à produit égal, un pays très inégalitaire n'atteindra pas le même niveau de population stationnaire.

Bref, conclut Quetelet!: «!quand on veut établir des comparaisons entre les peuples, il est de la plus grande importance de consulter la qualité, si je puis m'exprimer ainsi, autant que la quantité!». Ces idées seront reprises quelques années plus tard par Ernst Engel dans ses études sur le niveau de vie et l'évolution des dépenses de consommation. Pour bien marquer la filiation de ces travaux, Engel y proposait d'ailleurs, en hommage à Quetelet, de retenir le terme «!Quet!» comme nom de l'unité de mesure du niveau de vie.

Comme on le voit, la thèse de Quetelet, tout en prolongeant les intuitions de Malthus, semble enrichir considérablement la théorie développée par ce dernier et poser

\footnotetext{
${ }^{37}$ Sur l'homme..., op. cit., p. 250.

${ }^{38}$ Ibidem, p. 251-252.

${ }^{39} \mathrm{Sa} \ll$ !population spécifique!» pour reprendre la terminologie de Quetelet.

${ }^{40}$ Ibidem, p. 253.

${ }^{41}$ Ibidem, p. 256 (C'est nous qui soulignons, B.D.).
} 
les bases, dès 1835 , d'un modèle formel d'analyse de la croissance à long terme de la population qui était censé permettre aux démographes et aux économistes de prévoir l'évolution comparée de la population et des ressources disponibles.

Cependant, de manière assez surprenante, cet exposé tourne court. Quetelet ne donne aucune équation précisant son théorème de résistance, il ne fournit même aucun graphique, ce qui étonne, alors qu'équations et graphiques émaillent le texte sur des points auxquels il accordait pourtant beaucoup moins d'importance. Bien plus, quand il en vient à l'étude de deux cas concrets -!la population de l'Angleterre de 1700 à 1830 et celle des Etats-Unis de 1780 à 1825!- il combine, dans le calcul des formules de croissance, des phases de croissance linéaire et des phases de croissance exponentielle sans utiliser de terme de freinage. Ce sont là, selon toute vraisemblance, les indices d'une difficulté insurmontée dans la mise en œuvre par Quetelet de l'«!analogie mécanique!» en matière de théorie de la population. Nous aurons à revenir ultérieurement sur les causes de ces lacunes et de ces difficultés, en comparant les approches de Quetelet et de Verhulst, mais la «!tentative de greffe de concepts mécaniques!» ${ }^{42}$ en démographie semble en définitive peu probante.

\section{LA MODÉLISATION MATHÉMATIQUE DE VERHULST}

\section{L'ITINÉRAIRE D'UN DISCIPLE}

Verhulst est un proche de Quetelet, celui-ci y insiste dans sa nécrologie!: «!il fut successivement mon élève et mon collaborateur!; mon collègue à l'École militaire et mon confrère à l'Académie!; je m'honore d'avoir été son ami, depuis l'instant où j'ai pu le connaître jusqu'à celui où la mort nous a séparés! ${ }^{43}$.

Quetelet avait été en effet le professeur de mathématiques de Verhulst à l'Athénée Royal de Bruxelles et un tel enseignant eut vraisemblablement une grande influence sur la décision du disciple de poursuivre des études de mathématiques à l'Université de Gand (où Quetelet avait, lui aussi, obtenu son doctorat quelques années auparavant). Il y excella au point de remporter, dans l'année de ses vingt ans, un prix de l'Université de Leyde pour un mémoire sur la théorie des maxima et des minima et la médaille d'or de la Faculté des Sciences de Gand pour un travail sur le calcul des variations. Il soutint sa thèse de docteur ès sciences sur la résolution algébrique des équations binômes à 21 ans.

De retour à Bruxelles, il entama sa collaboration avec son maître, en particulier dans le cadre de ses contributions à la revue Correspondance mathématique et physique, fondée par Quetelet et Garnierr. ${ }^{44}$ C'est dans ce cadre que Quetelet contribua à faire «!descendre le jeune géomètre de la région des abstractions sur le terrain de l'arithmétique politique!» ${ }^{45}$. C'était à la fois dans l'intérêt de ce jeune collaborateur, puisque, dans cette voie «!il montrait une aptitude toute particulière!» mais, de plus, «!il pouvait être utile [...] d'avoir quelques hommes qui fussent en état d'examiner et d'approfondir les questions d'intérêt général en rapport avec les sciences

\footnotetext{
${ }^{42}$ Pour reprendre la formule utilisée par Giorgio Israel à propos d'un autre modèle de dynamique des populations, celui de Volterra (voir La mathématisation du réel, op. cit., p.66).

${ }^{43}$ Quetelet, «!Notice sur Pierre-François Verhulst [...]!», p. 26-30.

${ }^{44}$ Verhulst était chargé de la relecture des épreuves de la revue, ce dont Quetelet le remercie à l'occasion. Garnier avait été le professeur de mathématiques de Quetelet puis de Verhulst à l’Université de Gand.

${ }^{45}$ Quetelet, «!Notice sur P.-F. Verhulst!», op. cit., p. 11.
} 
mathématiques!» ${ }^{46}$. Ainsi, «!il se mit à étudier la théorie des probabilités, et à se rendre familier les principes de l'économie politique!; il se trouvait là sur un terrain nouveau, mais encore peu ferme et mal limité!; il pouvait y donner large carrière à son goût pour la discussion!; aussi ce genre d'études devint-il pour lui l'objet d'une passion nouvelle!». ${ }^{47}$ Il rédigea ainsi - loutre ses mémoires sur la loi d'accroissement de la population!- un mémoire sur les loteries, et aida Quetelet dans ses travaux sur les tables de mortalité.

Mais ces études ne lui suffisaient pas, il cherchait un travail de plus longue haleine. Dissuadé par Poisson de s'attaquer à une édition complète des œuvres d'Euler, Quetelet lui propose de collaborer à une traduction du Traité de la lumière de John Herschel, qu'il souhaitait entreprendre. Ce fut Verhulst qui assura finalement seul cette tâche, Quetelet se chargeant de la rédaction des notes et d'un supplément.

Si l'on excepte une courte collaboration comme enseignant de mathématiques aux cours publics organisés au Musée de Bruxelles en $1829^{48}$, sa carrière de professeur débuta vraiment en 1834 à l'École militaire. C'est dans ce cadre qu'il entreprit de rédiger un Traité élémentaire des fonctions elliptiques, manuel qui mettait à la portée des étudiants les travaux de Legendre, Abel et Jacobi. Cette œuvre, publiée en 1841, lui ouvrit la même année les portes de la section des sciences de l'Académie royale de Belgique.

Cette consécration obtenue à la fin de sa - courte - vie ne doit cependant pas faire illusion!: alors que Quetelet remplira sans discontinuer des fonctions officielles ou académiques très importantes qui le contraindront à une certaine réserve, Verhulst, lui, aura une vie assez mouvementée, très engagée en tout cas dans les combats politiques et sociaux qui agitent la Belgique à cette époque.

Malheureusement pour lui, il manque l'épisode essentiel de la révolution de Bruxelles. ${ }^{49}$ Cet été 1830 le trouve en effet en Italie où il soigne, sous des cieux plus cléments, une grave tuberculose $^{50}$. Qu'à cela ne tienne, le combat pour la liberté n'ayant pas de frontières pour lui, il se lance aussitôt dans un projet grandiose!: «!opérer une réforme dans les États pontificaux et![...] persuader le Saint-Père de donner une constitution à son peuple!» ${ }^{51}$. Il en vient même à «!jouer le rôle d'un abbé Sieyès au petit pied!», comme il le reconnaît quelque temps plus tard avec beaucoup d'humour et de clairvoyance rétrospective ${ }^{52}$, puisqu'il va jusqu'à rédiger lui-même le projet de constitution, qu'il soumet pour amendements à la reine Hortense, alors à Rome, avant de le présenter aux cardinaux. «!Hélas, lui dit la reine, si le Pape était homme à faire les concessions convenables, il serait demain le chef de toute l'Italie. Il dicterait peut-être encore des lois à l'Europe, et rendrait à la religion, alliée à la liberté, la splendeur

\footnotetext{
${ }^{46}$ Ibidem.

${ }^{47}$ Ibidem. Comme Quetelet le précise plus loin, «!moins exclusif que la plupart des écrivains politiques, il accordait une égale importance à la statistique et à l'économie sociale!», ibidem, p. 22.

${ }^{48} \mathrm{Il}$ y avait bien sûr été recommandé par Quetelet qui y enseignait la physique.

${ }^{49}$ Qui éclate, comme l'on sait, le 25 août 1830 à l'issue de la représentation de l'opéra de Auber la Muette de Portici.

${ }^{50}$ Quetelet - décidément obnubilé par l'«!homme moyen!»!!!- attribue dans sa biographie cet état maladif à l'excès de travail et surtout «!à un développement de taille peu ordinaire!: sa hauteur était de $1,89 ! \mathrm{m}$ ou de 6 pieds environ!», «!Notice sur P.-F. Verhulst!», op. cit., note 1 p. 12.

${ }^{51}$ Quetelet, ibidem, p. 13.

${ }^{52}$ Voir la lettre de Verhulst dans l'édition du matin de L'indépendant (Bruxelles) du 29 novembre 1833, $\mathrm{n}^{\circ} 333,3^{\mathrm{e}}$ année, p. 1-2.
} 
qu'elle avait autrefois. Mais, en conscience, croyez-vous que ces bons religieux comprendront un mot de ce que vous allez leur dire!? Chaque changement leur paraîtra un sacrilège! ${ }^{53}$. La reine avait raison. Elle put heureusement le prévenir à temps que, faute de rencontrer les cardinaux (qui étaient tombés des nues en prenant connaissance du projet), il risquait fort d'avoir affaire à leurs sbires, qu'ils s'apprêtaient à lui envoyer. Il put ainsi s'enfuir de justesse des États pontificaux et revenir en Belgique.

De retour à Bruxelles, après une courte participation à l'organisation... logistique de l'armée belge pendant la résistance à l'invasion de l'armée hollandaise en 1831, son activité devient intense ${ }^{54}$ et se développe selon trois axes!:

- Une tentative d'action politique directe!: il lance un appel -!infructueux!- aux électeurs pour devenir membre de la Chambre des Représentants. Ceci lui vaut des critiques de Quetelet, pour qui peu de savants et d'artistes

\begin{abstract}
se sont distingués dans l'arène parlementaire, et [...] beaucoup au contraire y on perdu leur réputation. [...] La plupart du temps, l'Etat perd des hommes éminents dans leur spécialité. [...] La véritable source de leur talent, l'imagination, au lieu de s'exercer et de se développer dans les assemblées délibérantes, finit au contraire par s'émousser et s'éteindre en présence d'intérêts presque constamment matériels et sur lesquels portent les discussions 55 .
\end{abstract}

- Une œuvre réformatrice et civique illustrée notamment par la rédaction de deux opuscules!:

- un mémoire sur les abus dans l'enseignement supérieur actuel et sur les moyens de les réformer ${ }^{56}$, qui critiquait la dégradation de l'état des universités sous le pouvoir hollandais.

- un texte patriotique, le Précis historique des troubles de Bruxelles en $1718^{57}$, où Verhulst «!relisait l'histoire nationale [de la Belgique] et y recherchait avidement les époques qui pouvaient offrir quelque analogie avec celle où le pays se trouvait alors!» ${ }^{58}$.

- Mais surtout il exerça - jusqu'à sa mort précoce en 1849 - la fonction de «!maître des pauvres!» à la «!commission pour l'amélioration de la condition des pauvres dans les Flandres!» et participa à la commission sur les «!assurances générales par l’État!».

\footnotetext{
${ }^{53}$ Mémoires de la reine Hortense, tome III, p. 211. Selon le professeur Jean Mawhin - actuel président de l'Académie Royale de Belgique - le projet de constitution sera publié le 15 juillet 1831 dans le quotidien français Le National, qui l'attribuera «!au prêtre belge Verluest (sic)!» et Mawhin, qui manie aussi bien l'autodérision que les mathématiques ajoute avec humour!: «!d'aucuns diront qu'il fallait être surréaliste comme un belge, naïf comme un mathématicien et exalté comme un phtisique pour concevoir l'idée de doter les États pontificaux d'une constitution démocratique!» (J. Mawhin, op. cit.). Il faut préciser cependant que ce texte ne présente pas un contenu révolutionnaire très marqué!: s'il promeut l'instauration des libertés et de la séparation des pouvoirs, il restreint le suffrage à «!tous ceux qui, pour la conservation de leur fortune, sont intéressés à la stabilité du gouvernement!» (Lettre de Verhulst à L'indépendant, p. 1).

${ }^{54}$ Toujours partisan de la litote, Quetelet susurre, à propos de l'activité politique de Verhulst en Belgique!: «!C'est dire assez qu'il ne demeura pas étranger aux événements qui s’y passait alors!»!! (op. cit., p. 14).

${ }^{55}$ Quetelet, «!Notice sur P.-F. Verhulst!», op. cit., p. 15.

${ }^{56}$ Bruxelles, Hayez, 1831. Curieusement, Quetelet n'évoque absolument pas cet opuscule dans la bibliographie pourtant quasi-exhaustive des travaux de Verhulst!!

${ }^{57}$ Bruxelles, Th. Lejeune, 1832.

${ }^{58}$ Quetelet, «!Notice sur P.-F. Verhulst!», op. cit., p. 15.
} 
Verhulst faisait montre, on le voit, d'une volonté d'action sur le terrain politique et social sans commune mesure avec l'activité publique de Quetelet, délibérément limitée à la promotion de l'Observatoire de Bruxelles tout d'abord, puis à celle des institutions et sociétés savantes de statistique.

Enfin, autre différence majeure avec son maître!:

il se montrait [...] peu favorable à l'application de la théorie des probabilités aux phénomènes moraux, et particulièrement aux déductions a posteriori que l'on tire de l'observation des faits. Il était, à cet endroit d'une réserve et d'une timidité qu'on pouvait regarder comme extrêmes : il ne jugeait le calcul applicable que quand on aperçoit un rapport direct entre la cause et l'effet. Sa conviction paraissait cependant ébranlée, quand on lui parlait des tables de mortalité et des applications diverses qui en ont été faites, même dans ses propres écrits ${ }^{59}$.

\subsection{DE LA LOI DE QUETELET AU MODÈLE DE VERHULST.}

Tel est le collaborateur auquel Quetelet décide de soumettre sa loi d'accroissement de la population!: «!Je priai M. Verhulst de soumettre ce principe à un calcul approfondi et d'en faire l'application aux meilleurs documents connus sur la population!» ${ }^{60}$. Bien qu'exprimée à mots couverts, ce passage trahit bien la difficulté rencontrée par Quetelet!: il ne s'agit pas seulement de «!vérifier!» la validité de la loi en l'appliquant «!aux meilleurs documents connus!» mais aussi «!de soumettre à un calcul approfondi!» le principe de résistance proportionnelle au carré de la vitesse de croissance de la population. Quetelet est manifestement bloqué dans sa tentative de transcription en démographie des lois de la résistance aérodynamique.

S'il fallait un autre indice de cette difficulté, on le trouverait dans un fragment de note de Quetelet, conservé dans les archives de l'Académie royale, où celui-ci discute les thèses de Verhulst en ramenant le problème du freinage de la croissance à des arguties sur les paramètres des équations physiques de la chute des corps pesants, appelant à la rescousse l'autorité - certes prestigieuse - de Siméon-Denis Poisson et de son Traité de mécanique ${ }^{61}$.

\footnotetext{
${ }^{59}$ Ibidem, p. 21-22. Et Quetelet poursuit!: «!Vers la fin de sa vie, il revint encore sur sa thèse favorite, et me confia qu'il méditait, pour notre séance publique [de l'Académie], un discours dans lequel il entreprenait de combattre mes idées sur la théorie des probabilités appliquée à la statistique morale!; il craignait que sa santé ne lui permit pas de le lire!; j'offris d'en donner communication à sa place, disant que sa réfutation en serait d'autant plus piquante. Cette idée lui souriait, mais il n'eut ni le temps ni la force de donner suite à ce projet!».

${ }^{60}$ Quetelet, «!Notice sur P.-F. Verhulst!», op. cit., p. 20.

${ }^{61}$ Archives de l'Académie royale, fonds Quetelet (Les passages entre crochets sont barrés dans le manuscrit), Liasse $n^{\circ} 153$ !: [!fragment relatif à la!] «!Note de M. Verhulst!» p. 14!:

«!(1) Ceci serait vrai si l'on admettait avec l'auteur que le coefficient $\mathbf{m}$, dans les formules qu'il donne $\mathrm{d}$ 'après $\mathrm{M}$. Poisson, conserve une valeur constante, comme dans la chute des corps à travers une [petite] couche d'air assez petite pour pouvoir être considérée comme un fluide homogène!; mais les formules et les résultats seraient différents, si le corps tombait d'une grande hauteur, car $\mathbf{m}$ varierait à raison du changement de densité des couches de l'atmosphère!: Poisson, ibidem p. 300. C'est aussi de cette manière que nous avons considéré les choses!; car il est évident que si, dans un même lieu et l'état social ne changeant pas [d'ailleurs], la population croissait à l'infini on ne pourrait plus regarder $\mathbf{m}$ comme constant et les formules citées ne seraient plus applicables. Pour ne laisser aucun doute à cet égard, nous citions l'exemple du nuage qui monte ou retombe jusqu'à une certaine limite, selon sa densité, sa température, la direction des vents et une foule d'autres circonstances atmosphériques. «!!Ce qui n'empêche cependant pas qu'il revienne toujours à une certaine hauteur moyenne, dépendant de sa
} 
Le travail de Verhulst sur la dynamique des populations donna lieu à quatre publications d'importance inégale!:

- une «!Notice sur la loi que la population suit dans son accroissement!» de 9 pages, publiée en 1838 dans le tome $\mathrm{X}$ de la Correspondance mathématique et physique [référencée «!Notice!» ci-dessous]!;

- un premier mémoire de 39 pages illustré d'un graphique, lu à la séance de l'Académie du 30 novembre 1844 et paru sous le titre «!Recherches mathématiques sur la loi d'accroissement de la population!» dans le tome XVIII (1845) de la série des Nouveaux mémoires de l'Académie Royale des Sciences et Belles-Lettres de Bruxelles [ci-dessous!: «! ${ }^{\mathrm{er}}$ Mémoire »!].

- une texte de 3 pages!: «!Note sur la loi d'accroissement de la population [...]!» paru en 1846 dans les Bulletins de l'Académie Royale des Sciences et BellesLettres de Bruxelles, au $\mathrm{n}^{\circ} ! 4$ du tome XIII [ci-dessous!: «!Note!»].

- enfin un!: «!Deuxième mémoire sur la loi d'accroissement de la population!» de 32 pages, lu à la séance de l'Académie du 15 mai 1846 et paru en 1847 dans le tome XX de la série des Mémoires de l'Académie Royale des Sciences des Lettres et des Beaux-Arts de Belgique, [ci-dessous!: «!2 ${ }^{\mathrm{e}}$ Mémoire!»],

De manière générale, on peut dire que les réactions de Quetelet à ces publications oscillent entre les demi-vérités et les malentendus profonds. C'est le cas en particulier pour la «!Notice!» qui est analysée ainsi par Quetelet dans la nécrologie de Verhulst!:

Notre collègue voulut bien se prêter à ma demande, et publia les résultats de ces recherches dans le tome $X$ de la Correspondance mathématique et physique. Il fut conduit à cette conclusion, que les données de l'observation étaient encore trop peu nombreuses pour que le principe énoncé put être vérifié, de manière à ne laisser aucun doute sur son exactitude; ou, en d'autres termes, que la théorie avait devancé l'observation et qu'il était prudent de s'arrêter ${ }^{62}$.

L'examen attentif de la «!Notice!» amène à des conclusions moins anodines. Citons en effet le texte de Verhulst!:

On peut faire diverses hypothèses sur la résistance ou la somme des obstacles opposés au développement indéfini de la population. M. Quetelet la suppose proportionnelle au carré de la vitesse avec laquelle la population tend à croître. C'est assimiler le mouvement de la population à celui d'un mobile qui tombe en traversant un milieu résistant. Les résultats de cette comparaison s'accordent, d'une manière satisfaisante, avec les données de la statistique et avec ceux que j'ai obtenus par mes propres formules, quand on suppose aux couches de milieu traversé, une densité indéfiniment croissante $^{63}$.

constitution et de l'obstacle qu'il trouve à sa chute par la résistance de l'air!», Physique sociale, t. 1, p. 280 (p. 252 de la réédition 1991). Nous n'avons pas pu avoir en vue ce que deviendrait la population par la suite des siècles si elle se trouvait condensée outre mesure à la surface du globe!; nous n'avons entendu parler que pour des limites de temps restreintes et nous sommes heureux de voir que, d'après les calculs de M. Verhulst, notre hypothèse satisfait aux observations. Quand on voudra étendre les prévisions plus loin, il faudra avoir égard à la variabilité de $\mathbf{m}$, cas dans lequel le corps [tombant] peut demeurer suspendu et stationnaire dans le milieu par lequel il tombait d'abord, nous avons lieu de croire qu'alors se trouvera la même concordance entre les résultats des calculs et ceux d'observation. [Les lois de la population telles que nous les avons posées ne sont au surplus qu'une extension des lois généralement admises pour une autre classe de phénomènes]!».

${ }^{62}$ Quetelet, «!Notice sur P.-F. Verhulst!», op. cit., p. 20.

${ }^{63}$ Verhulst, «!Notice!», p. 114. 
La «!loi de Quetelet!» n'a donc en aucun cas pour Verhulst le statut de loi de la nature, elle y est présentée au contraire comme une hypothèse parmi d'autres. Si l'on en juge par la fin de la citation précédente, et par le fragment transcrit ci-dessus à la note 60 (qui semble se référer à ce passage), l'attitude de Verhulst est même plus subtile puisqu'elle consiste à prendre Quetelet en défaut sur son propre terrain. En substance, son message est le suivant!: «!d'accord pour une application du principe physique de la résistance à la traversée d'un milieu dense, toutefois un tel problème ne répond pas à une seule loi mais à une batterie de relations mathématiques bien différentes selon que la densité est stable ou croissante, que la température est plus ou moins élevée...!».

Par politesse sans doute, Verhulst attribue cependant à la théorie de son maître un certificat de conformité aux données, mais c'est pour préciser aussitôt que ses propres formules donnent également de bons résultats. Verhulst n'a donc pas décidé de s'arrêter sur la voie de la théorisation en attendant de pouvoir tabler sur un ensemble de données satisfaisant, comme le prétend Quetelet. Sa position est, là encore, plus subtile, elle est bifide!:

- Certes, dit-il, «!j'ai tenté depuis longtemps de déterminer par l'analyse, la loi probable de la population!; mais j'ai abandonné ce genre de recherches parce que les données de l'observation sont trop peu nombreuses pour que les formules puissent être vérifiées, de manière à ne laisser aucun doute sur leur exactitude» ${ }^{64}$.

- Mais faute de pouvoir atteindre cette «!véritable loi!» de la population, Verhulst propose une nouvelle démarche qui diverge fondamentalement de celle de Quetelet!:

- L'objectif essentiel n'est pas de prédire de manière précise le profil de croissance de la population d'un pays, mais de déterminer la population maximale qu'elle peut atteindre compte tenu de son évolution passée et de son niveau actuel de civilisation, «!ce maximum sera le chiffre de la population devenue stationnaire! ${ }^{65}$.

- De ce point de vue, on peut essayer diverses fonctions retardatrices!; elles donnent toutes sensiblement les mêmes résultats. Leur formule générale peut être exprimée en faisant apparaître la vitesse de croissance comme une fonction décroissante du niveau atteint par la population!:

$$
\frac{d p}{d t}=(\mathrm{m} \cdot \mathrm{p})-\square(\mathrm{p})
$$

où $m$ représente le taux de croissance instantané dans une situation où la croissance ne rencontre aucun obstacle, $t$ le temps, $p\left(\right.$ ou $\left.p_{t}\right)$ l'effectif de la population au temps $t$, et $\square(p)$ une certaine fonction de $p$ à spécifier.

L'approche est, comme on le voit, très différente de celle de Quetelet!: il s'agit, pour la première fois dans ce contexte, de tester une batterie de modèles mathématiques sans rechercher l'adéquation parfaite au processus que seule «!la loi véritable!» - au statut de loi physique - pourrait fournir, mais en se bornant à s'assurer qu'elles vérifient!:

\footnotetext{
${ }^{64}$ Ibidem, p. 115.

${ }^{65}$ Ibidem.
} 
1) la condition fondamentale sur l'issue du processus!: «!Toutes les formules par lesquelles on essaiera de représenter la loi de la population, doivent [...] satisfaire à la condition d'admettre un maximum qui ne soit atteint qu'à une époque infiniment éloignée. Ce maximum sera le chiffre de la population devenue stationnaire! ${ }^{66}$.

2) une conformité suffisante avec les données disponibles, que Verhulst -!tout comme Quetelet!- sait d'ailleurs bien peu fiables encore à l'époque, malgré leurs efforts d'amélioration et de correction.

Verhulst indique ainsi avoir testé successivement pas moins de quatre fonctions retardatrices ${ }^{67} !$ :

$$
\square(\mathrm{p})=\mathrm{n} \cdot \mathrm{p}^{2} \quad \square(\mathrm{p})=\mathrm{n} \cdot \mathrm{p}^{3} \quad \square(\mathrm{p})=\mathrm{n} \cdot \mathrm{p}^{4} \quad \square(\mathrm{p})=\mathrm{n} \cdot \log (\mathrm{p})
$$

les résultats étant proches, il suffit selon lui de retenir «!l'hypothèse la plus simple que l'on puisse faire!» ${ }^{68}$, c'est-à-dire !:

$$
\square(\mathrm{p})=\mathrm{n} \cdot \mathrm{p}^{2}
$$

L'expression de la vitesse de croissance est alors!:

$$
\frac{d p}{d t}=(\mathrm{m} \cdot \mathrm{p})-\left(\mathrm{n} \cdot \mathrm{p}^{2}\right)
$$

d'où l'on déduit, en intégrant (1)!:

$$
\mathrm{t}=\frac{1}{m}[\log (\mathrm{p})-\log (\mathrm{m}-\mathrm{np})]+\mathrm{c}
$$

et, en résolvant par rapport à $p$ :

$$
\mathrm{p}=\frac{\mathrm{m} \cdot \mathrm{p}_{0} \cdot \mathrm{e}^{\mathrm{m} \cdot \mathrm{t}}}{\mathrm{n} \cdot \mathrm{p}_{0} \cdot \mathrm{e}^{\mathrm{m} \cdot \mathrm{t}}+\mathrm{m} \square \mathrm{n} \cdot \mathrm{p}_{0}}
$$

En faisant tendre le temps $t$ vers l'infini, on déduit de cette équation générale la valeur de $\boldsymbol{P}$, la «!limite supérieure de la population!»!:

$$
\mathbf{P}=\frac{m}{n}
$$

Peut-être par excès de prudence, Verhulst ne donne dans la «!Notice!» que les tableaux permettant de comparer, pour quatre zones, les données officielles avec les populations calculées grâce à la formule (3) sans fournir, ni les paramètres calculés dans chaque cas, ni surtout l'effectif de la population maximale, mais il est facile pour nous de les retrouver à partir des tableaux et de la méthode de calcul détaillée dans le premier mémoire.

En suivant pas à pas les indications de l'auteur, nous trouvons ainsi par exemple pour la France la formule!:

\footnotetext{
${ }^{66}$ Ibidem.

${ }^{67}$ Où $n$ est une constante à déterminer d'après les données.

${ }^{68}$ Ibidem, p. 115.

${ }^{69}$ Le symbole $c$ représente la constante d'intégration.

${ }^{70}$ Où $p_{0}$ est la population en $\mathrm{t}=0$ et $e$ la base des logarithmes népériens. Ibidem, p. 116.
} 


$$
\mathrm{p}=\frac{0,0314 \square 29,981 \square \mathrm{e}^{0,0314 . t}}{\left(0,000813 \square 29,981 \square \mathrm{e}^{0,0314 . t}\right)+0,0314 \square(0,000813 \square 29,981)}
$$

si la population est exprimée en millions et le temps en nombre d'années depuis 1817 (année qui correspond à $\mathrm{t}=0$ !). Les paramètres correspondant au tableau donné par Verhulst sont en effet approximativement!:

$$
\mathrm{m}=0,0314 \quad \mathrm{n}=0,000813
$$

et l'on peut déduire la population maximale de la France!: $\mathbf{P}=\frac{\mathrm{m}}{\mathrm{n}} \square 38,6$ millions

Verhulst introduit certes en conclusion quelques nuances mais, de manière assez surprenante, la difficulté est imputable selon lui à la faible qualité des données utilisées, qui ne permettent pas de vérifier l'exactitude de sa formule. Si, du fait de cette limite, «!l'avenir seul pourra nous dévoiler le véritable mode d'action de la force retardatrice que nous avons représentée par $\square(p) !\rangle^{71}$, il reste convaincu de l'intérêt de sa démarche!:

- «!les résultats auquel je suis parvenu peuvent offrir de l'intérêt au moins comme objet de spéculation!»!;

- «!la marche que j'ai suivie me paraît devoir conduire nécessairement à la connaissance de la véritable loi!» ${ }^{72}$.

Si l'accent est mis ici essentiellement sur l'intérêt heuristique de ses travaux, on constate que Verhulst prétend ne pas renoncer à l'objectif essentiel, la recherche de «!la loi véritable de la population!». Le jour où la fiabilité des données statistiques permettrait, comme dans les sciences physiques, de mesurer les «!erreurs!» des modèles, c'est-à-dire l'écart entre données calculées et données observées, on pourrait espérer en tirer partie pour déterminer la «!véritable loi!».

Verhulst ajoute in fine une précision d'allure anodine!: «!mon travail était terminé en 1833 , et je n'y ai plus touché depuis!». Remarque de détail, introduite peut-être simplement pour justifier le caractère relativement ancien des données utilisées, mais qui peut aussi être une manière discrète d'indiquer que ses propres recherches sont antérieures à celles dont Quetelet rend compte dans son ouvrage Sur l'homme et le développement de ses facultés.

\subsection{LES ENJEUX ÉPISTÉMOLOGIQUES DE LA MODÉLISATION}

Au-delà de l'anecdote et de la complexité des relations - concurrentielles autant qu'amicales - entre les deux académiciens, il est clair que les deux approches épistémologiques sont sensiblement différentes. Il semble même, au vu de leurs premiers textes respectifs, que l'on puisse appliquer au couple Quetelet!-!Verhulst, le diagnostic très clair posé par Giorgio Israel sur l'opposition entre Volterra -!attaché à un point de vue strictement déterministe et mécaniste - et Lotka, partisan, lui, de l'utilisation des analogies mathématiques. Si le débat est plus récent de presque un siècle, le champ scientifique est par ailleurs très proche (est-ce un hasard!?). C'est, dans

\footnotetext{
${ }^{71}$ Ibidem, p. 116 (c'est nous qui soulignons, B.D.).

${ }^{72}$ Ibidem, p. 115.
} 
le cas de Volterra et de Lotka, celui de la dynamique comparée des populations de proies et de prédateurs.

Il suffit presque de relire ici les développements qu'y consacre Giorgio Israel dans La mathématisation du réel, en remplaçant le nom de Volterra par celui de Quetelet et le nom de Lotka (ou de Van der Pol) par celui de Verhulst pour voir combien le schéma s'applique bien ${ }^{73}$. Qu'on en juge!:

[L'exemple de Volterra-Lotka] nous permet de comprendre combien il était difficile pour un savant classique (mécaniste traditionaliste comme Volterra) d'accepter le procédé de l'analogie mathématique - cette espèce de danse sur l'abîme qui, avec de la chance, peut conduire à des réussites inattendues, mais peut aussi mener à des désastres que seuls les procédés méthodiques et prudents du réductionnisme classique peuvent nous épargner.

Mais c'est précisément le saut de l'acrobate au-dessus de l'abîme qui peut permettre de passer d'une discipline à une autre, même très éloignée, et d'établir des connections et des liens inattendus. En ce sens, l'application des mathématiques aux sciences non physiques résulte surtout de l'analogie mathématique.

L'analogie mécanique peut assurément ouvrir des voies nouvelles, comme l'exemple de Volterra le montre clairement ${ }^{74}$. [...] Mais le ralliement pur et simple au schéma mécanique, l'imitation précise du modèle des sciences physico-mathématiques ne peuvent pas permettre d'aller bien loin. En certains cas, elles peuvent produire une véritable " scolastique ${ }^{75}$.

Une approche telle que celle de Van der Pol ${ }^{76}$ est l'expression d'une vision plus «libre » (orientée vers l'axiomatisation) sans un système de règles a priori. Depuis le début du XX $X^{e}$ siècle [...], l'essor impétueux de la modélisation mathématique, conduisit principalement à cette vision sans pour autant la débarrasser complètement de l'influence de la mécanique! $!^{77}$.

L'application des mathématiques à l'extérieur du cercle de la physique ne commence certes pas au XX ${ }^{\mathrm{e}}$ siècle. Mais selon Israel le seul exemple au XVIII ${ }^{\mathrm{e}}$ est celui de la théorie mathématique de la variole et de l'utilité de l'inoculation proposée par Daniel Bernoulli. Quant aux modélisations tentées au cours du XIX ${ }^{\mathrm{e}}$ siècle - surtout en statistique, démographie et biométrie - elles «!ont une portée assez modeste, souvent en raison de leur niveau mathématique très bas!: 1 ' «!intensité!» de la mathématisation y est si faible que les mathématiques restent à l'arrière-plan des structures théoriques en jeu!» ${ }^{78}$. La modélisation mathématique ne prendra vraiment son essor qu'au début du siècle suivant, à l'occasion d'un «!processus d'invasion des mathématiques dans des domaines traditionnellement considérés comme relevant de l'histoire ou d'une approche

\footnotetext{
${ }^{73}$ Si G. Israel présente de manière limpide le modèle de Verhulst (op. cit., p. 89-92) dans le cadre de la visite de son «!zoo des modèles mathématiques!», il ne le compare malheureusement pas dans cet ouvrage à la loi de Quetelet (peut-être le fait-il ailleurs, nous ne le savons pas).

${ }^{74} \mathrm{G}$. Israel précise ici que «!Le grand paradoxe dans ce domaine, est que le seul "modèle" scientifique de référence est celui de la physique. Il est difficile, pour le chercheur qui aborde la biologie ou l'économie, de recourir à des schémas ou à des cadres conceptuels différents de ceux qui sont offerts par les sciences physico-mathématiques, en particulier par la mécanique!».

75 «!Tel est le cas de la tentative de Volterra pour bâtir une sorte de «!mécanique analytique!» des associations biologiques qui suit strictement l'exemple de la mécanique hamiltonienne!» (note de G. Israel).

${ }^{76}$ Inventeur en 1926 d'un modèle mathématique décrivant les battements du cœur.

${ }^{77}$ G. Israel, op. cit., p. 200.

${ }^{78}$ Ibidem, p. 211.
} 
purement expérimentale (comme les sciences sociales et économiques ou les sciences du vivant). La mathématisation de ces domaines, que les spécialistes, en accord parfait avec les physiciens et les mathématiciens, avaient séparés strictement des mathématiques, est un phénomène caractéristique de notre siècle, qui est loin d'être achevé!» ${ }^{79}$.

Si l'on s'interroge sur les causes de la pauvreté des résultats obtenus par la mathématisation de la biologie et de l'économie ${ }^{80}$, l'une des principales est sans doute, selon Giorgio Israel, que la modélisation mathématique du monde non physique inclut «!la vieille ambition, toujours vivante et agissante, de réaliser une description mécanique et réductionniste unifiée du monde, une ambition qui résiste à toutes les difficultés et à tous les échecs!» ${ }^{81}$. À la différence du réductionnisme classique qui postule l'unité et l'objectivité du savoir scientifique et qui considère le modèle comme une image mathématique unique et univoque de la réalité, il faudrait désormais le concevoir comme une construction ad hoc, une «!sonde conceptuelle!» parmi d'autres possibles.

Giorgio Israel résume ainsi sa thèse en indiquant que!: «!les caractéristiques spécifiques de la modélisation mathématique sont essentiellement au nombre de deux.

- En premier lieu, le renoncement à toute tentative d'aboutir à une image unifiée de la nature!: un modèle mathématique est un fragment de mathématique appliqué à un fragment de réalité. Non seulement un seul modèle peut décrire différentes situations réelles, mais le même fragment de réalité peut être représenté à l'aide de modèles différents.

- En second lieu, la méthode fondamentale de la modélisation est l'analogie mathématique (où le fragment de mathématiques unifie tous les phénomènes qu'il est censé représenter), et non plus l'analogie mécanique, qui a été pendant très longtemps le procédé principal de la mathématisation!» ${ }^{82}$.

Tous ces ingrédients se retrouvent bien dans le premier modèle de Verhulst et dans les arguments de son débat avec Quetelet, qui s'épuise, lui, à trouver le «!bon!» schéma mécanique applicable à la croissance de la population. Jusqu'aux repentirs mêmes - réels ou simulés - de Verhulst qui prétend garder pour objectif la découverte de «!la!» loi de croissance «!véritable!» - reportée cependant aux calendes grecques pour cause de faible sûreté des données disponibles. Les textes ultérieurs, par l'impression qu'ils donnent d'essais successifs d'application d'un fragment de mathématique au même fragment de réalité, ne feront, comme nous le verrons, que confirmer cette première impression.

\subsection{LA PREMIÈRE REPRÉSENTATION DE LA LOI LOGISTIQUE}

Le «!Premier mémoire!» publié sept ans plus tard reprend pour l'essentiel la méthode de la «!Notice!». Il commence par l'examen de l'hypothèse de croissance géométrique qui «!ne se réalise que dans des circonstances tout à fait exceptionnelles!; par exemple, quand un territoire fertile et d'une étendue en quelque sorte illimitée, se trouve habité par un peuple d'une civilisation très avancée!». Les statistiques démographiques des

\footnotetext{
${ }^{79}$ Ibidem, p. 201.

${ }^{80}$ Cf., G. Israel, op. cit., p. 327.

${ }^{81}$ Ibidem, p. 328.

${ }^{82}$ Ibidem, p. 11 (c'est nous qui soulignons, B.D.).
} 
Etats-Unis, seul pays de ce type où les données sont connues, permettent à Verhulst de vérifier la validité approximative de la loi de croissance exponentielle limite donnée par Malthus, atteignant un doublement tous les 25 ans!:

$$
\mathrm{p}_{\mathrm{t}}=\mathrm{p}_{0} \cdot \mathrm{e}^{\square . \mathrm{t}} \quad \text { avec, quel que soit } t !: \quad \mathrm{p}_{\mathrm{t}+25}=2 \cdot \mathrm{p}_{\mathrm{t}}
$$

ce qui correspond au taux de croissance instantané!:

$$
\square=\frac{\ln 2}{25}=0,02773 \approx 2,77 \%{ }^{83}
$$

Il corrige ensuite quelque peu la thèse de 1837 en indiquant que cette croissance exponentielle - qu'il appelle, lui, «!logarithmique!» - peut être observée pour toutes les populations tant qu'elles n'ont pas eu à connaître d'entraves à leur croissance. Tout peuple est dans ce cas tant qu'il n'a pas atteint sa «!population normale!» (notée $b$ par Verhulst). Ce niveau une fois atteint, le taux de croissance de la population n'est plus constant mais diminue au fur et à mesure que la population augmente. Le raisonnement est ici le même que dans la «!Notice!»!: «!On peut faire une infinité d'hypothèses sur la loi d'accroissement du coefficient [ $[$ ]. La plus simple consiste à regarder cet affaiblissement comme proportionnel à l'accroissement de la population, depuis le moment où la difficulté de trouver des bonnes terres a commencé à se faire sentir!» ${ }^{84}$. La relation retenue entre le taux de croissance instantané et l'effectif de la population est donc linéaire, elle peut être exprimée sous la forme suivante ${ }^{85}$ !:

$$
\square(\mathrm{t})=-\frac{\mathrm{a}}{\mathbf{P}} \cdot \mathrm{p}+\mathrm{a}
$$

ce qui correspond bien, pour ce qui concerne la relation entre $p$ et $t$ à la même fonction que celle de la «!Notice!». La formule est certes exprimée ici en fonction de la «!population surabondante!», c'est à dire de l'écart $p-b$, mais cela ne change rien à la forme mathématique de la relation retenue. D'ailleurs aucune précision n'est donnée sur la détermination possible du niveau de la «!population normale!» $b$ ni a fortiori sur la date où elle est atteinte.

Le mémoire insiste par ailleurs beaucoup sur les développements mathématiques de l'hypothèse initiale et sur les méthodes numériques permettant de calculer les paramètres de cette fonction, et il en donne une représentation graphique ${ }^{86}$. De plus, pour la première fois semble-t-il, il lui donne le nom qu'elle portera désormais, celui de «!fonction logistique!».

Finalement, en appliquant sa méthode aux données belges pour les années 1815, 1830 et 1845 , Verhulst trouve une population maximale de 6,6 millions d'habitants pour le royaume «!si les lois et les mœurs de la Belgique n'éprouvaient aucun changement notable! $\gg^{87}$. À partir des données française de 1818,1830 et 1842, il obtient -!sous les mêmes réserves de stabilité des lois et des mœurs!- une population maximale française de 40 millions d'habitants.

\footnotetext{
${ }^{83}$ Soit un taux de croissance annuel de $2,81 \%$ par an.

${ }^{84}$ «!Premier Mémoire!», p. 8.

${ }^{85}$ Où $a$ est une constante à déterminer et $\boldsymbol{P}$ la population maximale.

${ }^{86}$ Ibidem, p. 39 bis. Voir. infra Figure 2.

${ }^{87}$ Ibidem, p. 31.
} 
La conclusion du mémoire est intéressante sur le plan épistémologique, mais peu explicite en ce qui concerne la position propre de Verhulst sur la question du paupérisme!:

Quand on songe aux calamités que doit nécessairement amener l'exubérance toujours croissante de la population, et à l'insuffisance, bien avérée aujourd'hui, des moyens essayés par les modernes pour y remédier, on ne peut s'empêcher d'être frappé de cette réflexion d'Aristote, à propos de la République de Platon : "Peut-être serait-il d'une bonne politique, de fixer le nombre des enfants plutôt que celui des propriétés, et de permettre ou de restreindre les naissances, d'après des calculs basés sur la stérilité ou le nombre des morts. C'est l'imprévoyance des gouvernements sur un point aussi essentiel, qui peuple aujourd'hui nos cités de tant de misérables ; de là, tant de séditions et de crimes, dont la pauvreté est la mère ${ }^{88}$. Il faut que les maux dont il s'agit aient été bien vivement éprouvés par les anciens, pour qu'un de leurs plus illustres moralistes ${ }^{89}$, ait osé louer les pauvres d'exposer ou de détruire leurs enfants, dans la crainte de les élever pour l'indigence et la servilité : "car, dit-il, ils ne peuvent supporter l'idée de leur laisser pour héritage la pauvreté, qu'ils regardent comme le plus grand des maux, comme une grave et cruelle maladie ».

La loi de la population nous est inconnue, parce qu'on ignore la nature de la fonction qui sert de mesure aux obstacles, tant préventifs que destructifs, qui s'opposent à la multiplication indéfinie de l'espèce humaine.

Cependant, si l'on suppose que ces obstacles croissent exactement dans la même proportion que la population surabondante, on obtient la solution complète du problème, sous le point de vue mathématique.

On trouve alors, en faisant usage des documents statistiques publiés par les gouvernements belge et français, que la limite extrême de la population, est de quarante millions pour la France, et de six millions six cent mille âmes, pour la Belgique.

Une longue série d'observations, non interrompues par de grandes catastrophes sociales ou des révolutions du globe, fera probablement découvrir la fonction retardatrice dont il vient d'être fait mention ${ }^{90}$.

La réaction précise de Quetelet au mémoire n'est pas connue, mais l'on peut s'en faire une idée à partir d'un manuscrit de l'Académie royale!: le rapport préalable à la publication rédigé le 31 octobre 1844 par Dandelin ${ }^{91}$ et contresigné par Quetelet ${ }^{92}$. Le rapport commence par un point d'accord manifeste!:

Ce mémoire renferme des vues intéressantes, et parmi elles, la plus saillante c'est que quelle que soit la tendance à l'accroissement de la population, il se trouve à côté de cette tendance, une résistance dont l'origine tient à une foule de causes inconnues ou inobservées : cette résistance crô̂t en même temps que l'accroissement de la population en telle façon que la différence devient de plus en plus faible entre les gains et les pertes, et qu'en définitive, il y a un maximum de population qui ne peut être dépassé pour une localité donnée.

\footnotetext{
${ }^{88}$ Politique, livre II, chap. 4.

${ }^{89}$ Plutarque, De amore prolis, $\mathrm{V}$.

${ }^{90}$ Verhulst, op. cit., p. 37-38 (c'est nous qui soulignons, B. D.).

${ }^{91}$ Ancien professeur de mathématique de Verhulst à l'Université de Gand.

${ }^{92}$ Il note en marge!: «!je souscris entièrement aux conclusions de l'honorable rapporteur!».
} 


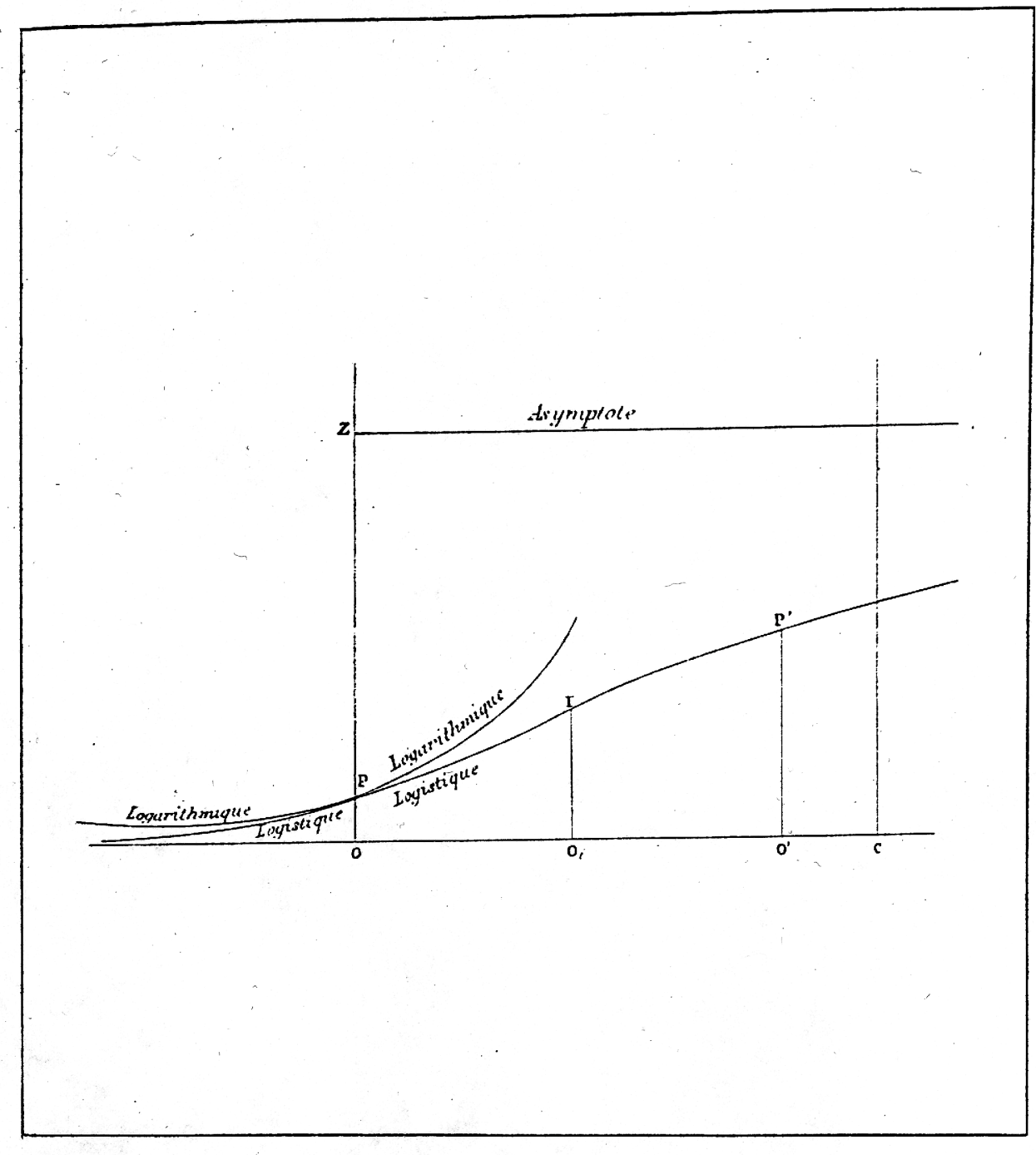

Mémorice sur la population par M. P. Yerhulst.

Figure 2. Représentation des fonctions «!logarithmique!» (i. e.!: exponentielle) et logistique dans le premier mémoire de Verhulst

Mais la fin du rapport est beaucoup moins élogieuse. Sans refuser l'imprimatur pour la publication dans la série des Mémoires de l'Académie, le rapport indique que le travail et la conception qui ont conduit aux calculs du maximum de population sont «!contestables en eux-mêmes et très éloignés de notre opinion sur cette importante matière!» ${ }^{93}$. C'est dire combien, malgré la proximité des idées de Verhulst, Dandelin et Quetelet sur les tendances d'évolution à long terme de la population, le fossé était grand entre les procédures de raisonnement considérées, de part et d'autre, comme valides.

\subsection{L'ABANDON DE LA LOGISTIQUE}

Le deuxième mémoire, bien que parvenant à des conclusions sensiblement différentes des textes précédents ne dévie pas de la ligne épistémologique essentielle choisie dès

\footnotetext{
${ }^{93}$ Académie royale, Correspondance Quetelet, liasse $n^{\circ} 007175$.
} 
1837. C'est en effet la volonté de l'auteur de perfectionner la forme mathématique de l'équation d'évolution chronologique du taux de croissance elle-même qui l'amène à une révision du modèle initial.

Le schéma général reste celui qui peut être exprimé, en termes de taux d'accroissement relatif sous la forme !:

$$
\frac{\mathrm{dp}}{\mathrm{dt}} \cdot \frac{1}{\mathrm{p}}=\square-\mathrm{f}(\mathrm{p}-\mathrm{b})
$$

Mais, ce taux d'accroissement relatif étant un nombre pur $^{94}$, il faudrait, selon l'auteur, que la fonction $f(p-b)$ corresponde également à un nombre pur. Il se reproche de ne point y avoir veillé dans son premier travail. En essayant, une fois encore, les fonctions «!par ordre de simplicité!»" ${ }^{95}$, le terme retardateur le plus simple satisfaisant aux conditions imposées est désormais proportionnel, non plus à la «!population surabondante!» $(p !-! b)$, comme dans le premier mémoire, mais au rapport de la population surabondante à la population totale, c'est-à-dire au terme!:

$$
\frac{\mathrm{p} \square \mathrm{b}}{\mathrm{p}}
$$

L'équation du taux d'accroissement relatif devient ainsi!:

$$
\frac{\mathrm{dp}}{\mathrm{dt}} \cdot \frac{1}{\mathrm{p}}=\square-\mathrm{k} \cdot \frac{\mathrm{p} \square \mathrm{b}}{\mathrm{p}}
$$

d'où l'on déduit une relation linéaire ${ }^{96}$ ! entre le temps et l'écart de la population courante ( $p$, exprimée en millions) à la population maximale $(P)$ !:

$$
\mathrm{t}=\frac{1}{\mathrm{~m}} \cdot \ln \frac{\mathrm{P} \square \mathrm{p}_{0}}{\mathrm{P} \square \mathrm{p}}
$$

Verhulst aboutit finalement, à partir de la comparaison des données de trois années équidistantes à une équation, plus simple que la logistique du mémoire précédent, dont la forme est maintenant une fonction de type exponentiel ${ }^{97}$, donnant la population $p$ en fonction de $t$. Par exemple pour la Belgique ${ }^{98}$ !:

$$
\mathrm{p}=\mathrm{P}-\frac{\mathrm{P} \square \mathrm{p}_{0}}{\exp (0,00751939 \square \mathrm{t})}=9,439-\frac{5,812}{\exp (0,00751939 \square \mathrm{t})}
$$

La population maximale déduite des données est ici de 9 millions $1 / 2$ d'habitants environ $(9,439)$ et la «!population normale!» est estimée à un peu plus de 2 millions d'habitants, ce qui correspond à l'année 1785. Avant cette date, on peut considérer, selon Verhulst, que la croissance est exponentielle et qu'elle se fait au taux constant limite évoqué par Malthus pour l'Amérique, c'est à dire un taux instantané de

\footnotetext{
${ }^{94}$ Un nombre «!abstrait!» dans la terminologie de Verhulst.

95 «!Deuxième mémoire!», p. 6.

${ }^{96}$ Le symbole $p_{0}$ correspond bien sûr à la population en $t_{0}$, l'origine des temps retenue!; $m$ est un coefficient à déterminer d'après les données étudiées.

${ }^{97}$ Elle est qualifiée de «!logarithmique!» (comme dans le cas de la croissance sans obstacles) dans la terminologie de Verhulst.

${ }^{98} \mathrm{Si}$ l'on compte les années à partir de $\mathrm{t}_{0}=1815$ avec $\mathrm{p}_{0}=3,627$. V. Schtickzelle, op. cit., p. 553.
} 
$2,7726 ! \%^{99}$. L'équation donnant $p$ en fonction de $t$ (pour $\mathrm{t}<1785$ ) est alors pour la Belgique!:

$$
\mathrm{p}=\exp [0,769182+0,02776(\mathrm{t}-1785)]
$$

On est ainsi passé, du premier au second mémoire, d'une courbe logistique unique à une courbe à deux phases se rejoignant à l'ordonnée de la «!population normale!». La Figure 3 ci-dessous que nous avons reconstruite à partir des hypothèses des deux textes permet de comparer les deux schémas d'évolution. On constate que les populations maximales diffèrent mais que l'allure générale est la même.

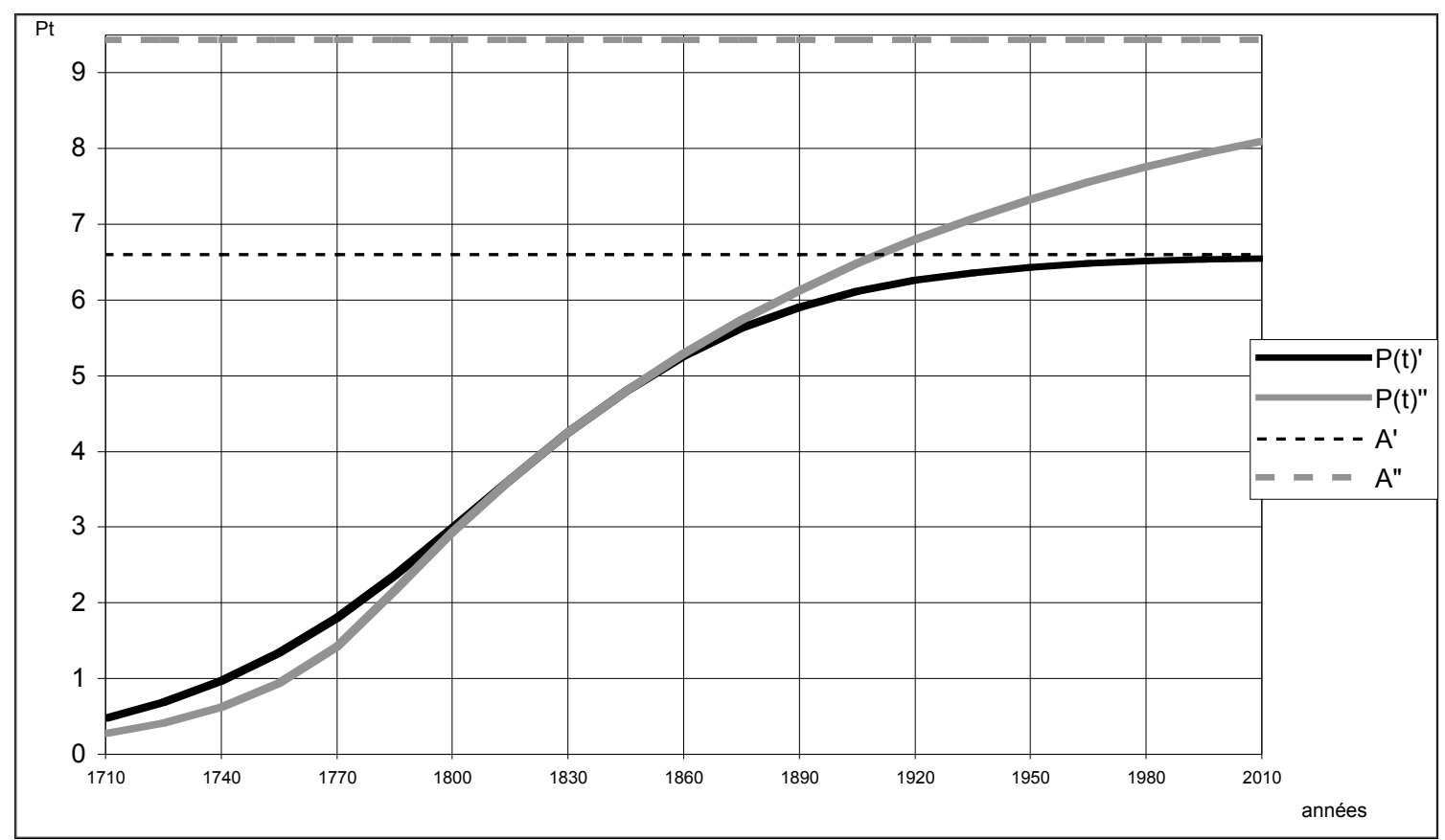

Figure 3. Comparaison de l'évolution de la population belge suivant le premier mémoire $\left(\mathrm{p}_{(\mathrm{t})}{ }^{\prime}\right.$, asymptote $\left.\mathrm{A}^{\prime}=6,6\right)$ et le second $\left(\mathrm{p}_{(\mathrm{t})}{ }^{\prime}{ }^{\prime}\right.$, asymptote $\left.\mathrm{A}^{\prime}{ }^{\prime}=9,44\right)$

Le deuxième, et dernier, mémoire sur la population apporte donc peu par rapport aux textes précédents, si l'on excepte la forte «!revalorisation!» de la population maximale, et la tentative de construction d'une sorte de «!corrélation des rangs!» ante litteram croisant pour l'ensemble des départements français le niveau d'instruction et la fécondité des mariages.

Le texte se termine par des considérations générales peu explicites sur le fameux débat fécondité-ressources. Les positions de Verhulst restent très vagues. Qu'on en juge!:

Pour attendre de malheureux [...] l'observation des préceptes les plus simples de la morale, il faudrait commencer par faire régner parmi eux un certain degré d'aisance. Or c'est là justement ce qui est impossible, à moins d'une demande de travail extraordinaire ou d'une diminution dans la

\footnotetext{
${ }^{99}$ Soit $\ln 2$ divisé par 25 (temps de doublement), comme on 1'a indiqué précédemment.
} 
population. Le genre humain tourne donc dans un cercle fatal, l'excès de la population engendrant la misère, et la misère entretenant à son tour l'excès de la population par son excessive fécondité ${ }^{100}$.

Quoique les observations précédentes semblent donner raison aux défenseurs de l'opinion pessimiste, il y aurait sans doute de la témérité à les prendre pour base d'un jugement définitif. Il est certain que le goût du luxe et du bien-être, en pénétrant de plus en plus dans les derniers rangs de la société, doit tendre à augmenter le nombre des célibataires et à diminuer la fécondité des mariages. Mais à notre avis, ce n'est pas moins une erreur de croire que la pauvreté cessera d'être le partage de l'immense majorité du corps social. Quelle que soit la part de plus en plus grande que les animaux et les machines doivent occuper dans l'industrie par suite des progrès de la science, cette majorité sera toujours condamnée à de pénibles travaux. Toujours il y aura des laboureurs, des mineurs, des matelots, des pêcheurs. Sans parler des obstacles provenant de la religion, peut-on raisonnablement attendre de gens voués à d'aussi rudes professions, qu'ils apportent dans l'union conjugale la prudence raffinée des fermiers bas-normands? Si des circonstances favorables venaient à rendre la misère moins commune ou moins poignante, peut-on croire que c'est alors qu'ils se soumettraient à des privations, à des gênes contre nature, auxquelles ils ne veulent point se plier aujourd'hui ? Et, dans le cas où la misère augmenterait, ce qui paraît être en ce moment celui de la Belgique, l'expérience de tous les siècles et de tous les pays a démontré que cette cause ne suffit pas pour engager les prolétaires à restreindre leur multiplication. Nous ne pouvons donc nous empêcher de penser que le sort des classes laborieuses n'est guère susceptible de s'améliorer, aussi longtemps qu'on n'aura pas trouvé un moyen purement artificiel de tenir constamment l'offre de la main d'ouvre au-dessous de la demande. C'est là l'énigme proposée aux économistes par ce sphinx des temps modernes qu'on appelle le paupérisme ${ }^{101}$.

Engagés sous le signe de la volonté de construire un grandiose modèle de synthèse de l'évolution à long terme de la population, les travaux de Verhulst semblent bien aboutir à une impasse. Après avoir combattu avec succès par ses recherches les opinions alarmistes prévoyant l'inéluctabilité de la croissance explosive du nombre de bouches à nourrir, l'auteur semble retomber dans une conception étriquée, celle d'une société enfermée dans un cercle vicieux où les progrès eux-mêmes peuvent devenir une cause de régression. Par comparaison, «!'’illustre Quetelet!», traditionaliste en matière épistémologique, apparaît ainsi comme beaucoup plus optimiste que son turbulent disciple sur les possibilités de progrès social réalisable grâce aux lumières de la physique sociale.

Il reste cependant, comme nous espérons l'avoir montré à partir de l'étude détaillée des textes originaux, que le débat entre le maître et le disciple dépassait de très loin une divergence de point de vue sur les possibilités de progrès de la société. Il manifeste un des aspects de l'opposition entre l'approche analogique et l'approche mécaniste avec ses enjeux différents selon les sciences, si bien décrite par Giorgio Israel!:

L'essor de la modélisation mathématique et de la démarche "analogique » est intimement lié à la crise du modèle unitaire et mécaniste de la réalité. La diffusion de l'approche modéliste en physique témoigne du déclin du rôle de la physique mathématique et de la mécanique classique. Elle se présente

\footnotetext{
100 «!Deuxième mémoire!», p. 21.

${ }^{101}$ Ibidem, p 31-32.
} 
comme un processus de crise, car quoi que l'on en dise, la physique s'est bâtie autour de l'aspiration à une image unitaire de la réalité. La modélisation représente donc un renoncement. La tradition historique de la physique s'accommode mal de l'idée que les mathématiques constituent seulement un réservoir d'images qu'on peut appliquer de diverses manières à la réalité ${ }^{102}$. À l'inverse, l'essor de la modélisation dans les sciences non physiques est tel que l'histoire de la modélisation mathématique coüncide avec une part importante de l'histoire de leur mathématisation. Preuve que l'élimination du schéma étroit et rigide du mécanisme a rendu possible une application sans obstacles des mathématiques, justement parce qu'elle n'est pas fondée sur la prétention de vouloir aboutir à une description unique de la réalité écrite en termes mathématiques. Les concepts des sciences non physiques ont été forgés presque toujours selon une démarche non mathématique, recourant parfois à des images mathématiques fournies par les modèles, mais sans s'y réduire ni se restreindre à un seul type de représentation, qui aurait sinon joué un rôle central et non auxiliaire ${ }^{103}$.

Parti d'une approche modeste de «!bricolage!» de fonctions susceptibles de rendre compte d'une croissance freinée, Verhulst est parvenu à une formulation élégante et simple de ce type de dynamique. Malheureusement, si le modèle fonctionne très bien pour interpoler voire pour «!rétropoler!» des séries fragmentaires ${ }^{104}$, il est beaucoup moins performant pour la prévision à moyen terme où trop de facteurs non contrôlés interviennent sur les résultats ${ }^{105}$. Les tentatives de généralisation ${ }^{106}$ sont restées, en tout cas dans le domaine des populations humaines, de faible intérêt pratique!: «!plus un modèle mathématique se complique pour mieux coller à la réalité, moins les mathématiques sont capables d'en donner les propriétés. L'information tirée d'un modèle varie en sens inverse de sa sophistication!» ${ }^{107}$.

\section{BIBLIOGRAPHIE}

N.B. : On a indiqué entre crochets, pour les ouvrages anciens, les cotes de la Koninklijke Bibliotheek-Bibliothèque Royale Albert $1^{\text {er }}(K B R)$ de Bruxelles.

\section{A) GUVRES DE VERHULST}

Commentatio de maximis et minimis [!en latin!], 1824, Luchtmans, Université de Leyde, 30 !pages. [!Cote BNF!: R 5313 (7)!].

\footnotetext{
${ }^{102}$ C'est nous qui soulignons, B. D.

${ }^{103}$ Giorgio Israel, La mathématisation du réel, op. cit., p. 81.

${ }^{104}$ Donc, en quelque sorte pour «!prévoir le passé!».

${ }^{105}$ C'est ce que relevait déjà le grand statisticien Ronald Aylmer Fisher en 1927 dans son commentaire de l'exposé de Pearl «!Biology of Population Growth!» à la conférence mondiale de la population de Genève où il concluait à la «!failure of the logistic!» et précisait ainsi son point de vue (p. 45, nous soulignons)!: «!Its general character however, is such to make it an admirable curve for interpolation over short ranges, and even over short-range prediction, provided we have not any other important relevant information!». «!In the case of human populations, we possess in the recorded age of individuals every relevant information, which is of even greater importance than the census totals, and enables us to make a prediction with very much more certainty than can be made from any curve of census totals [...]. When we have such information, it would be foolish to ignore it, and as the British population exemplifies, it may point to conclusions very different from those indicated by the logistic curve!».

${ }^{106}$ V. Mawhin, art. cit., p. 6-7.

${ }^{107}$ Ibidem, p. 7.
} 
De resolutione, tum algebraica, tum lineari aequationum binomalium [!en latin!], Thèse de mathématique [!Dissertatio mathematica inauguralis ], soutenue à Bruxelles, le 3 Août 1825, Université de Gand, 1825, 27 pages. [!Cote B.U. Lille 1!: 55 !776 / $1825 / 1$ ! ].

Traduction par P.-F. Verhulst du Traité de la lumière, de John Frederick William Herschel, avec notes de Quetelet!; Paris, Malher, 1829. [!Cote KBR!: $5^{\text {ème }}$ Cl. II Hers!; cote BNF!: V 41615-41616!].

Mémoire sur les abus dans l'enseignement supérieur actuel et sur les moyens de les réformer, Bruxelles, Hayez, 1831. [!BNF!: $8^{\circ} \mathrm{R}$. pièce 2233!].

Précis historique des troubles de Bruxelles, en 1718!; Bruxelles, Lejeune, 1832. [!Cote KBR!: II!86.932 / A / 4!].

«!Notice sur la loi que la population suit dans son accroissement!», Correspondance mathématique et physique ${ }^{108}$, tome X - ou tome II de la $3^{\mathrm{e}}$ série -, 1838, p. 113-121.

Traité élémentaire des fonctions elliptiques. Ouvrage destiné à faire suite aux traités élémentaires de Calcul intégral, Bruxelles, Hayez, 1841. [!Cote KBR!: 5 ${ }^{\mathrm{e}} \mathrm{Cl}$. I F $1 \mathrm{C}$ Verh!].

«!Recherches mathématiques sur la loi d'accroissement de la population!» [! ${ }^{\text {er }}$ Mémoire!], Nouveaux mémoires de l'Académie Royale des Sciences et Belles-Lettres de Bruxelles, tome XVIII, 1845. [!Cote KBR!: R 2355' / 18!; cote BNF!: R $5120(18,1)$ ou $4^{\circ} \mathrm{R}$ pièce 955 !].

«!Note sur la loi d'accroissement de la population», Bulletins de l'Académie Royale des Sciences et Belles-Lettres de Bruxelles, tome XIII, $\mathrm{n}^{\circ}$ 4, 1846, 3 pages

«!Deuxième mémoire sur la loi d'accroissement de la population!», Mémoires de l'Académie Royale des Sciences des Lettres et des Beaux-Arts de Belgique ${ }^{109}$, t.!XX, 1847, p.!1-32. [!Cote KBR!: R 2355 / 20!; cote BNF!: R 5120 (20,III)!].

\section{B) GUVRES DE QUETELET ${ }^{110}$}

Instructions populaires sur le calcul des probabilités, Bruxelles, Tarlier et Hayez, 1828. [!Cote KBR!: WBS VH 8.251 A [M-SLZ]!; reprint!: [KBR!: 9 A/1996/2.536!]!; reprint en fac simile (avec une introduction par Italo Scardovi et une bibliographie de Paola Geretto, Rome, Istituto centrale di statistica, 1996.

«!Notes extraites d'un voyage en Angleterre aux mois de Juin et Juillet 1833 !», in!: Correspondance mathématique et physique, op. cit.!; tome!8, 1835, p. 15-16.

Sur l'homme et le développement de ses facultés, essai de physique sociale, 2 vol., Bruxelles, 1835 [! $1^{\mathrm{e}}$ édition de la Physique sociale!]. Réédité - en 1 volume - dans la collection «!Corpus des œuvres de philosophie en langue française!», Paris, Fayard, 1991.

«!Sur l'appréciation des documents et en particulier sur l'appréciation des moyennes!», Bulletin de la Commission centrale de statistique [de Belgique]!, tome!II, 1844. [!KBR!: ARC A 4 P 129!].

\footnotetext{
${ }^{108}$ Cote KBR!: III 19979 A, disponible également à la bibliothèque Quetelet de Bruxelles.

${ }^{109}$ Les deux mémoires sont reliés ensemble dans!: «!P.-F. Verhulst, Opuscules!» cote KBR II 45979 A.

${ }^{110}$ N.B.!: La bibliothèque personnelle de Quetelet est accessible au Ministère des affaires économiques, Direction des études et de la documentation, 6 rue de l'industrie, Bruxelles, «!Bibliothèque Quetelet!».
} 
Lettres à S.A.R. le duc régnant de Saxe-Cobourg et Gotha, sur la théorie des probabilités appliquées aux sciences morales et politiques!, Bruxelles, Hayez, 1846. [!Cote BNF V 16904!].

Du système social et des lois qui le régissent!, Paris, Guillaumin, 1848. [!KBR!: ARC 8 B 00118 [M-ARC]!; BNF!: R 47 847!].

«!Sur la statistique morale et les principes qui doivent en former la base!», Mémoires de l'Académie Royale de Bruxelles, tome 21, 1848, 7. [!Cote KBR!: ARC 4 C 004 / 021 a [M-ARC]!:!cote BNF!: R 5120, XXI, 7!].

«!Notice sur Pierre-François Verhulst, membre de l'Académie!», Bruxelles, Hayez, 1850, avec portrait de Verhulst (reproduit en tête du présent article), p. 1-25, suivi de «!Publications faites par M. Verhulst!», p.!26-30. Extrait de l'Annuaire de l'Académie Royale de Belgique pour 1850. [!Cote KBR!: R 2355'].

Théorie des probabilités, Bruxelles, «!Encyclopédie populaire!», A. Jamar, (s. d.) [1853], in-16, 104 p., portrait [Cote BNF!: Z- 47850].

Histoire des sciences mathématiques et physiques chez les belges, Bruxelles, Hayez, 1864. [!Cote BNF!: V- 50357!].

Sciences mathématiques et physiques chez les belges au commencement du $19^{e}$ siècle, Bruxelles, Thiry-Van Buggenhoudt, 1866. [!Cote KBR!:II 18276 A!; BNF!: 509.034 QUET!].

[!Présentation en 8 pages, du $1^{\text {er }}$ vol. de la $2^{\mathrm{e}}$ édition de!:!] «!Physique sociale, sur l'homme et le développement de ses facultés, par Ad. Quetelet [...]!», Bulletins de l'Académie Royale de Bruxelles, tome XXVI, ${ }^{\circ} 12,1868^{111}$.

Physique sociale, ou essai sur le développement des facultés de l'homme, 2 vol., 1869 $\left[! 2^{\mathrm{e}}\right.$ édition de la Physique sociale!], Bruxelles, Muquardt!; Paris, Baillière!; St. Petersbourg, J.!Issakof [!KBR!: II 20018 A!]. Réédité avec annotations de Eric Vilquin et Jean-Paul Sanderson, Bruxelles, Académie Royale, 1997, XV-702 p.

\section{C) Articles de Raymond Pearl et Lowell J. Reed}

PEARL R. \& REED L.J., «!On the rate of growth of the population of the United States since 1790 and its mathematical representation!», Proceedings of the National Academy of Sciences (U.S.A.) 6(6), 1920, p. 275-288.

PEARL R. \& REED L.J., «!A further note on the mathematical theory of population growth!», Proceedings [...], op. cit., 8(10), 1922, p. 365-368.

PEARL R., «!The biology of death!», [the] Scientific Monthly 13, [série de trois articles dans le vol.!13], 1921!:

- «!The inheritance of duration of life in Man!», 13(1), july 1921, p. 46-66.

- «!Experimental studies on the duration of life!», 13(2), august 1921, p. 144-164.

- «!Public health and the population problem!», 13(3), sept. 1921, p.!193-213.

PEARL R. \& REED L.J., «!On the mathematical theory of population growth!», Metron 3(1), Rome, 1923, p. 6-19.

PEARL R., Studies in Human Biology, Williams and Wilkins Cy., Baltimore, 1924.

\footnotetext{
${ }^{111}$ Repris dans le recueil!: Varia [cote!: II 66000 A, vol. 1, p. 496 à 503] qui relie différents mémoires de Quetelet, dont celui-ci qui porte le $\mathrm{n}^{\circ} 55$.
} 
PEARL R., L'accroissement de la population au point de vue biologique, 25 p., reprinted from the Proceedings of the World Population Conference, Genève, 1927. [!Cote KBR!: III 80459 A, année 1927!; cote BNF!: $8^{\circ}$ T pièce 3596!].

PEARL R., Introduction to medical Biometry and Statistics, Philadelphie \& Londres, Saunders, $2^{\text {nd }}$ ed. 1930.

PEARL R., The natural History of Population, Oxford U. P., 1939.

\section{D) Autres ouvrages}

ACADEMIE ROYALE DE BELGIQUE, Adolphe Quetelet, 1796-1874, exposition documentaire présentée à la Bibliothèque royale Albert $\mathrm{I}^{\mathrm{er}}$, Bruxelles, Palais des Académies, 1974.

ARTZROUNI M., «!Une nouvelle famille de courbes de croissance. Application à la transition démographique!», Population 41(3), 1986, p. 497-510.

BAIR J., HAESBROECK G. et SALENGROS P., «!Modélisation de croissances décrites par une courbe sigmoïde!», Travaux de recherche du GEMME, n ${ }^{\circ}$ 9804, Université de Liège, 1998.

COLLARD A., «!Goethe et Quetelet. Leurs relations de 1829 à 1832!», Isis 20(2), Rome, 1933, p.426-435.

DELEVSKY J., «!Une formulation mathématique de la loi de la population!», Metron 7(4), Rome, 1928, p.75-96. [!Cote KBR!: R 4725!].

DE PAEPE J.-L. (éd.), Inventaire des Archives de l'Académie Royale de Belgique, Bruxelles, Académie Royale de Belgique, 1986.

DUPAQUIER J., «!Avez-vous lu Malthus!?», Population 35(2), 1980, p. 279-290.

DUPAQUIER J. et DUPAQUIER M., Histoire de la démographie, Paris, Librairie Académique Perrin, 1985.

FIRCKS A.F. von, Bevölkerungslehre und Bevölkerungspolitik, (Hand und Lehrbuch der Staatswissenschaften, I.!Abteilung [ $1^{\mathrm{e}}$ Partie]!: «!Volkswirtschaftslehre!», VI.!Band [Tome 6]), Leipzig, Hirschfeld, 1898.

HANOTEAU J. (éd.), Mémoires de la Reine Hortense [de Beauharnais], éditées par le Prince Napoléon, Paris, Plon, 1927.

HECHT J., «!Imagination et prospective!: les origines de la prévision démographique!», Mohammed RASSEM (ed.), Statistik und Staatbeschreibung in der Neuzeit, actes du colloque de Wolfenbüttel, 1978, Paderborn et al. loc., F. Schöning, 1980.

ISRAEL G., La mathématisation du réel. Essai sur la modélisation mathématique, Paris, éditions du Seuil, 1996.

JAMES P., Population Malthus, Londres, Routledge \& Kegan Paul, 1979.

JOURNAL OF THE ROYAL STATISTICAL SOCIETY 88, Jan. 1925, Londres, série d'articles sur la croissance de la population!:

- «!The Growth of Population and the Factors which control it!», G.!Udny Yule, p. 1-58.

- «!Proceedings of the Meeting!»!: interventions de Edgeworth, Athelstane Baines et réponse de Yule, p. 58-62.

- «!The Laws Governing Population!», par T.H.C. Stevenson, p. 62-76. 
- «!Discussion on Dr. Stevenson Governing Paper!»!: interventions de A.L. Bowley, William Bevedridge, Marie Stopes, Dr. Greenwood, G. U. Yule, p. 76-90.

KNAPP G.F., «!A. Quetelet als Theoretiker!», Jahrbücher für Nationalökonomie und StatistikI, 18, 1872, p. 89-124.

KINGSLAND S.E., Modeling Nature. Episodes in the History of Population Ecology, The University of Chicago Press, 1985.

LANDAU D. \& LAZARSFELD P., «!Quetelet, Adolphe!», International Encyclopaedia of the Social Sciences, vol.!XIII, New York, MacMillan, 1968, p. 247257.

LAZARSFELD P., Philosophie des sciences sociales, Paris, Gallimard, 1970.

LECUYER B.-P., «!!Quetelet!», Encyclopaedia Universalis, Paris, 1988.

LIAGRE J.-B. J., Calcul des probabilités et théorie des erreurs avec applications aux sciences d'observation en général et à la géodésie en particulier, 1879, $2^{\mathrm{e}}$ éd., Bruxelles, C. Muquardt, Merzbach \& Falk!; Paris, Gauthier-Villars, 592 p.

LOTTIN J., Quetelet, statisticien et sociologue, Louvain, Institut supérieur de philosophie, 1912.

MAILLY N. E., «!Essai sur la vie et les ouvrages de L.-A.-J. Quetelet!», Annuaire de l'Académie Royale de Belgique pour 1875, 41, p. 109-297.

MAWHIN J., «!Les héritiers de Pierre-François Verhulst!: une population dynamique!», Bulletin de la Classe des Sciences de l'Académie Royale de Belgique, [à paraître], (aimablement communiqué par l'auteur).

PELSENEER J., «!Pierre-François Verhulst!», Biographie Nationale, publiée par l'Académie Royale de Belgique, Bruxelles, tome 26, 1936-38, p. 658-663.

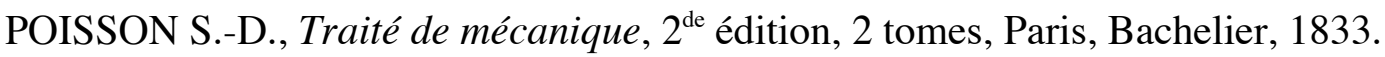

ROSENTAL P.-A., L'intelligence démographique, sciences et politiques des populations en France (1930-1960), Paris, éditions Odile Jacob, 2003.

ROSSI P., Cours d'économie politique, tome 1, $21^{\mathrm{e}}$ leçon!: «!Principe de la population et conclusion!», Paris, Joubert et Thorel, 1840, p. 386-419.

SAUVY A., «!La population stable et la population stationnaire selon Laplace et Quetelet!», Population 31(2), 1976, p. 485-487.

SCHTICKZELLE M., «!La première découverte de la fonction logistique!», Population 36(3), 1981, p. 541-556.

TOMASSONE R., LESQUOY É. et MILLIER C., La régression, nouveaux regards sur une ancienne méthode statistique, Paris, Masson, col. INRA, 1983.

VARII AUCTORES!: Mémorial A. Quetelet, publié à l'occasion du centième anniversaire de sa mort, Bruxelles, 1974. [!Cote KBR!: $2355^{\text {a }} \mathrm{R} / 33$ / 11!].

WELLENS-DE DONDER L., «!La Correspondance d'Adolphe Quételet!», Bruxelles, Centre d'histoire des sciences, 1964, extrait de!: Archives et bibliothèques de Belgique 35(1), 1964, p.!49-66. [!KBR, Département des manuscrits!: A 265 Mss (n 16)!] (Comprend une courte biographie de Quetelet).

WELLENS-DE DONDER L., Inventaire de la correspondance de Quetelet déposée à l'Académie Royale de Belgique, Bruxelles, Académie Royale, Classe des Sciences, Mémoires, Collection in $8^{\circ}$, tome 37, fasc.!2, 1966, 300 p. + gravures. [!KBR, Département des manuscrits!: A 988 Mss!]. 